\title{
VECTOR-VALUED EXTENSIONS FOR FRACTIONAL INTEGRALS OF LAGUERRE EXPANSIONS
}

\author{
ÓSCAR CIAURRI AND LUZ RONCAL
}

\begin{abstract}
We prove some vector-valued inequalities for fractional integrals defined in several contexts of orthonormal systems of Laguerre functions. On one hand, we obtain weighted $L^{p}-L^{q}$ vector-valued extensions, in a multidimensional setting, for the negative powers of the operator related to the so-called Laguerre expansions of Hermite type. On the other hand, we give necessary and sufficient conditions for vector-valued $L^{p}-L^{q}$ estimates related to the negative powers of the Laguerre operator associated to expansions of convolution type, in a one dimensional setting. Both types of vector-valued inequalities are based on estimates of the kernel with precise control of the parameters involved. As an application, mixed norm estimates for the fractional integrals related to the harmonic oscillator are deduced.
\end{abstract}

\section{INTRODUCTION}

The aim of this paper is the extension of $L^{p}-L^{q}$ mapping properties concerning fractional integrals (or negative powers, or potential operators) related to two second order differential operators of Laguerre type. More specifically, our target will be the proof of vector-valued extensions of some results given in [20]. We will deal with vector-valued inequalities of the form

$$
\left\|\left(\sum_{j=0}^{\infty}\left|T_{j} f_{j}\right|^{s}\right)^{1 / s}\right\|_{L^{q}(X, d \mu)} \leq C\left\|\left(\sum_{j=0}^{\infty}\left|f_{j}\right|^{s}\right)^{1 / s}\right\|_{L^{p}(X, d \mu)},
$$

where $\left\{T_{j}\right\}_{j \geq 0}$ is a sequence of operators defined on a measure space $(X, d \mu)$. We will also consider weighted vector-valued inequalities.

We first contextualize our problem. Let $I_{\sigma}$ be the fractional integral in the Euclidean space. The classical Hardy-Littlewood-Sobolev inequality (see, e.g., [11, 26]) establishes that

$$
\left\|I_{\sigma} f\right\|_{L^{q}\left(\mathbb{R}^{n}, d x\right)} \leq C\|f\|_{L^{p}\left(\mathbb{R}^{n}, d x\right)},
$$

when $1<p<\frac{n}{\sigma}$ and $\frac{1}{q}=\frac{1}{p}-\frac{\sigma}{n}$. Moreover, in the case $(p, q)=\left(1, \frac{n}{n-\sigma}\right)$ a weak type inequality holds. A weighted version of the Hardy-Littlewood-Sobolev inequality was given in [27].

The literature concerning the study of analogues of $I_{\sigma}$ is very vast. We are going to refer only a small part of the works dealing with fractional integrals associated with orthogonal expansions, and in particular Hermite and Laguerre expansions. Namely, B. Bongioanni and J. L. Torrea [4] obtained estimates for the negative powers of the multidimensional harmonic oscillator. Later, a most complete study of potential operators in the latter setting was developed in the work by A. Nowak and K. Stempak [21], where qualitatively sharp estimates for the potential kernel are obtained and $L^{p}-L^{q}$ boundedness of the potential operators is characterized. The boundedness from $L^{p}$ into itself of fractional integrals for Laguerre systems in dimension one was analyzed in [5], see also [15]. Fractional integrals for the multidimensional Laguerre expansions have been treated in [20]. There, the authors analyzed $L^{p}-L^{q}$ estimates (with and without weights) for the expansions related to

THIS PAPER HAS BEEN ACCEPTED IN: Studia Math..

2010 Mathematics Subject Classification. Primary: 42C10. Secondary: 47G40, 26A33, 42B35, 33C45.

Key words and phrases. Fractional integral, Laguerre expansions, vector-valued inequalities, weighted inequality, mixed-norm spaces.

The research of both authors is supported by grant MTM2015-65888-C04-4-P from Spanish Government. The second author is also supported by Basque Government through the BERC 2014-2017 program and by Spanish Ministry of Economy and Competitiveness MINECO: BCAM Severo Ochoa excellence accreditation SEV-2013-0323. 
Laguerre functions of Hermite type and Laguerre functions of convolution type (this nomenclature is used by S. Thangavelu in [29]). Sharp bounds for the one-dimensional kernel of potential operators in the case of the Laguerre functions of convolution type have been obtained in [22]. Concerning recent results in other settings: for instance, a complete and exhaustive study of fractional integrals for Jacobi and Fourier-Bessel expansions has been developed in [18]; moreover, a vector-valued extension in the Jacobi case was done in [8]. Finally, in [23], sharp $L^{p}-L^{q}$ results for potential operators are obtained in the framework of the twisted Laplacian and so-called special Hermite expansions.

If we denote, respectively, by $\left(L_{\alpha}^{H}\right)^{-\sigma}$ and $\left(L_{\alpha}\right)^{-\sigma}$ the fractional operators for Laguerre expansions of Hermite type and for Laguerre expansions of convolution type (see Section 2 for definitions), we are interested in the analysis of vector-valued inequalities for the sequences of operators $\left\{\left(L_{\alpha+a j}^{H}\right)^{-\sigma}\right\}_{j \geq 0}$ and $\left\{\left(L_{\alpha+a j}\right)^{-\sigma}\right\}_{j \geq 0}$, where $a$ is a positive real parameter. The meaning of $\alpha+a j$ will be explained below.

For Laguerre expansions of Hermite type we will consider the space $\left(\mathbb{R}_{+}^{n}, d x\right)$, with $d x$ the Lebesgue measure and $\mathbb{R}_{+}^{n}=(0, \infty)^{n}, n \geq 1$, and our approach will be similar to [20]. But in our case, we will prove an estimate for the kernels of the operators $\left(L_{\alpha+a j}^{H}\right)^{-\sigma}$ independent of the parameter $j$. Then a general result of vector-valued extensions will do the work. We will also include some potential weights.

On the other hand, we will deal with fractional integrals related to Laguerre expansions of convolution type, where the space considered will be $\left((0, \infty), d \mu_{\alpha}\right)$ with $d \mu_{\alpha}(r)=r^{2 \alpha+1} d r$. In this case, we provide necessary and sufficient conditions for the vector-valued extensions. However, the way to prove the corresponding vector-valued inequality will be close to the ideas in [4]. Observe that in [4], the authors consider Hermite expansions. The argument given in [20] to treat the convolution type setting is based on a very useful convexity principle. Unfortunately, we cannot apply the convexity principle here. The reason is the following: the constants appearing in this case involve Gamma functions whose log-convexity makes these constants increase with no control at all. The new estimates given in [22] for $\left(L_{\alpha}\right)^{-\sigma}$ are not appropriate for our target either: these estimates, applied to $\left(L_{\alpha+a j}\right)^{-\sigma}$, yield constants depending on the parameter $j$ and in that case we could not deduce the vector-valued extension.

Even more, the potential kernel of convolution type is dominated by the potential kernel of Hermite type (see (5.4)). This could be used to get $L^{p}-L^{q}$ estimates from the ones obtained in the Hermite case, but it happens that an additional condition on the parameters is needed, see the detailed explanation in Section 5. To avoid that restriction, suitable estimates with control on the parameters involved have to be proved.

At this point we notice that, unlike the case of the Laguerre expansions of Hermite type, the case of Laguerre expansions of convolution type is developed here in one dimension. For this case, the technical complexity to get the results is already high even in one dimension. The analysis in higher dimensions is more involved and we have not carried it out. Moreover, the important contribution in our work consists of a careful and thorough tracking of the constants involved that is noteworthy by itself, even in one dimension.

Well-known transference arguments exposed in [1] could easily imply analogous results to Theorem 2.1 and Theorem 2.3 for other Laguerre families. One may think that our weighted results in the multidimensional Hermite type setting (Theorem 2.1) imply the results in the convolution type setting (Theorem 2.3) by these transference arguments among Laguerre families, or reciprocally. Nevertheless, unlike the multidimensional Hermite type context, in the one dimensional convolution type context we provide necessary and sufficient conditions for the vector-valued extensions. It happens that the range of the parameter $q$ depending on $p$ in Theorem 2.1 (the multidimensional result) is not optimal. The reason is that, in the proof, we are estimating the corresponding kernel by a convolution kernel and a theorem by L. Hörmander ([16, Theorem 1.1]) states that nontrivial convolution operators cannot be bounded from $L^{p}\left(\mathbb{R}^{n}\right)$ to $L^{q}\left(\mathbb{R}^{n}\right)$ for $p>q$. See also the discussion in [20, p. 190]. Actually, an application of the transference arguments of [1] to the one dimensional unweighted version of Theorem 2.1 does not yield Theorem 2.3, in which the conditions on the parameters involved are proved to be sharp. Conversely, the multidimensional result in Theorem 2.1 cannot be obtained 
either by transference from Theorem 2.3 because the latter is proved only in one dimension. All in all, both Theorems 2.1 and Theorem 2.3 are of different nature and one does not imply the other by transference.

The proofs of the main results are based on the idea of representing the modified Bessel function through an integral, in which the integrated expression is symmetrized by means of a suitable change of variable. This idea, used by E. Sasso (see e.g. [25]), is presented with full detail, for instance, in [19].

As an application of our result on Laguerre expansions of convolution type, we will analyze the fractional integral operator related to spherical eigenfunctions of the harmonic oscillator. Observe that the result in [4] deals with eigenfunctions of the harmonic oscillator in cartesian coordinates. In our situation we consider the eigenfunctions obtained by using spherical coordinates (this is the reason for the name spherical eigenfunctions). In the spherical case, the eigenfunctions are products of Laguerre functions and spherical harmonics. We consider the mixed norm spaces $L^{p, 2}\left(\mathbb{R}^{n}, r^{n-1} d r d \omega\right)$ (see Section 3 for definition). These spaces were first sistematically studied by A. Benedek and R. Panzone in [3]. They arise frequently in harmonic analysis when the spherical harmonics are involved, see $[24,9,6,2,7]$.

The organization of the paper is the following. In Section 2 we introduce some definitions related to Laguerre systems and establish our results about boundedness of the associated fractional integrals. Section 3 contains our analysis of the fractional integrals for the harmonic oscillator in mixed norm spaces. In Section 4 and Section 5 we give the proofs of the results given in Section 2. Finally, in Section 6 we show the proofs of some technical results used along the paper.

\section{DEFINITIONS AND MAIN RESUltS}

We will first introduce some well-known facts concerning Laguerre functions.

Let $\alpha_{i}>-1, i=1, \ldots, n$ and $x=\left(x_{1}, \ldots, x_{n}\right) \in \mathbb{R}_{+}^{n}$. We define the first type of Laguerre functions $\varphi_{k}^{\alpha}(x)$ by

$$
\varphi_{k}^{\alpha}(x)=\varphi_{k_{1}}^{\alpha_{1}}\left(x_{1}\right) \cdots \varphi_{k_{n}}^{\alpha_{n}}\left(x_{n}\right)
$$

where $\varphi_{k_{i}}^{\alpha_{i}}\left(x_{i}\right)$ are the one dimensional Laguerre functions

$$
\varphi_{k_{i}}^{\alpha_{i}}\left(x_{i}\right)=\left(\frac{2 \Gamma\left(k_{i}+1\right)}{\Gamma\left(k_{i}+\alpha_{i}+1\right)}\right)^{1 / 2} L_{k_{i}}^{\alpha_{i}}\left(x_{i}^{2}\right) x_{i}^{\alpha_{i}+1 / 2} e^{-x_{i}^{2} / 2}, \quad x_{i}>0, \quad i=1, \ldots, n
$$

and $L_{k_{i}}^{\alpha_{i}}$ denotes the Laguerre polynomial of degree $k_{i} \in \mathbb{N}$ and order $\alpha_{i}>-1$, see [17, p. 76]. Moreover, the system $\left\{\varphi_{k}^{\alpha}\right\}_{k \in \mathbb{N}^{n}}$ is an orthonormal basis of $L^{2}\left(\mathbb{R}_{+}^{n}, d x\right)$. We will deal with the differential operator given by

$$
L_{\alpha}^{H}=-\Delta+|x|^{2}+\sum_{i=1}^{n} \frac{1}{x_{i}^{2}}\left(\alpha_{i}^{2}-\frac{1}{4}\right) .
$$

The operator $L_{\alpha}^{H}$ is symmetric and positive in $L^{2}\left(\mathbb{R}_{+}^{n}, d x\right)$ and the Laguerre functions $\varphi_{k}^{\alpha}(x)$ are eigenfunctions of (2.1). Indeed, we have $L_{\alpha}^{H} \varphi_{k}^{\alpha}=(4|k|+2|\alpha|+2 n) \varphi_{k}^{\alpha}$. By $|\alpha|$ and $|k|$ we denote $|\alpha|=\alpha_{1}+\cdots+\alpha_{n}$ (thus $|\alpha|$ may be negative) and the length $|k|=k_{1}+\cdots+k_{n}$. We will refer to $\varphi_{k}^{\alpha}$ as Laguerre functions of Hermite type.

For each $\sigma>0$, the fractional integrals for expansions in Laguerre functions of Hermite type are given by

where

$$
\left(L_{\alpha}^{H}\right)^{-\sigma} f=\sum_{m=0}^{\infty}(4 m+2|\alpha|+2 n)^{-\sigma} P_{m} f
$$

$$
P_{m} f=\sum_{|k|=m} a_{k}^{\alpha}(f) \varphi_{k}^{\alpha}, \quad a_{k}^{\alpha}(f)=\int_{\mathbb{R}_{+}^{n}} f(x) \varphi_{k}^{\alpha}(x) d x .
$$

With these notations, our result related to the fractional integrals for the Laguerre expansions of Hermite type is the following. 
Theorem 2.1. Let $\alpha \in[-1 / 2, \infty)^{n}$. Let $a \geq 1, \sigma>0,1<p \leq q<\infty, 1 \leq r \leq \infty, t<n / p^{\prime}$, $s<n / q, t+s \geq 0$.

(i) If $\sigma \geq n / 2$, then there exists a constant depending only on $\sigma$ and $\alpha$ such that

$$
\left\|\left(\sum_{j=0}^{\infty}\left|\left(L_{\alpha+a j}^{H}\right)^{-\sigma}\left(f_{j}\right)\right|^{r}\right)^{1 / r}\right\|_{L^{q}\left(\mathbb{R}_{+}^{n},|x|^{-s q} d x\right)} \leq C\left\|\left(\sum_{j=0}^{\infty}\left|f_{j}\right|^{r}\right)^{1 / r}\right\|_{L^{p}\left(\mathbb{R}_{+}^{n},|x|^{t p} d x\right)}
$$

for all $f_{j} \in L^{p}\left(\mathbb{R}_{+}^{n},|x|^{t p} d x\right)$.

(ii) If $\sigma<n / 2$, then the same boundedness holds under the additional condition

$$
\frac{1}{q} \geq \frac{1}{p}-\frac{2 \sigma-t-s}{n} .
$$

Remark 2.2. In the previous theorem, the sequence $\alpha+a j$ has to be understood as $\left(\alpha_{1}+a j, \ldots, \alpha_{n}+\right.$ $a j)$. It will be clear after the proof that this sequence can be changed into $\left(\alpha_{1}+a_{1}(j), \ldots, \alpha_{n}+a_{n}(j)\right)$ where $\left\{a_{i}(j)\right\}_{j \geq 0}$, for $i=1, \ldots, n$, are positive, increasing and unbounded sequences such that $a_{i}(0)=$ 0 and $a_{i}(1) \geq 1$.

Let us focus on the second setting. As explained in the introduction, we will consider only the one-dimensional context. Let $\alpha \geq-1 / 2$. We define another type of Laguerre functions $\psi_{k}^{\alpha}$ by (observe the change of notation: we use now $r$ for the one-dimensional variable)

$$
\psi_{k}^{\alpha}(r)=\left(\frac{2 \Gamma(k+1)}{\Gamma(k+\alpha+1)}\right)^{1 / 2} L_{k}^{\alpha}\left(r^{2}\right) e^{-r^{2} / 2}, \quad r>0 .
$$

These functions are related to the differential operator given by

$$
L_{\alpha}=-\frac{d^{2}}{d r^{2}}+r^{2}-\frac{2 \alpha+1}{r} \frac{d}{d r}
$$

which is a symmetric operator on $(0, \infty)$ equipped with the measure

$$
d \mu_{\alpha}(r)=r^{2 \alpha+1} d r
$$

The functions $\psi_{k}^{\alpha}$ form an orthonormal basis of $L^{2}\left((0, \infty), d \mu_{\alpha}\right)$ and are eigenfunctions of the differential operator (2.2). Indeed, we have $L_{\alpha} \psi_{k}^{\alpha}=(4 k+2 \alpha+2) \psi_{k}^{\alpha}$. We will refer to the functions $\psi_{k}^{\alpha}$ as Laguerre functions of convolution type.

For the expansions of Laguerre functions of convolution type we define the fractional integrals as

$$
\left(L_{\alpha}\right)^{-\sigma} f=\sum_{m=0}^{\infty}(4 m+2 \alpha+2)^{-\sigma} \mathcal{P}_{m} f
$$

where $\sigma>0$ and

$$
\mathcal{P}_{m} f=b_{m}^{\alpha}(f) \psi_{m}^{\alpha}, \quad b_{m}^{\alpha}(f)=\int_{0}^{\infty} f(r) \psi_{m}^{\alpha}(r) d \mu_{\alpha}(r) .
$$

Our result in this case is the following.

Theorem 2.3. Let $0<\sigma<\alpha+1, \alpha \geq-1 / 2, a \geq 1$ and $1 \leq p, q, s \leq \infty$. Define $u_{j}(r)=r^{a j}$, $r \in(0, \infty), j=0,1, \ldots$ Then there exists a constant depending only on $\sigma$ and $\alpha$ such that

$$
\left\|\left(\sum_{j=0}^{\infty}\left|u_{j}\left(L_{\alpha+a j}\right)^{-\sigma}\left(u_{j}^{-1} f_{j}\right)\right|^{s}\right)^{1 / s}\right\|_{L^{q}\left((0, \infty), d \mu_{\alpha}\right)} \leq C\left\|\left(\sum_{j=0}^{\infty}\left|f_{j}\right|^{s}\right)^{1 / s}\right\|_{L^{p}\left((0, \infty), d \mu_{\alpha}\right)}
$$

for all $f_{j} \in L^{p}\left((0, \infty), d \mu_{\alpha}\right)$, if and only if $p$ and $q$ satisfy

$$
\frac{1}{p}-\frac{\sigma}{\alpha+1} \leq \frac{1}{q}<\frac{1}{p}+\frac{\sigma}{\alpha+1},
$$

with exclusion of the cases $p=1$ and $q=\frac{\alpha+1}{\alpha+1-\sigma}$, and $p=\frac{\alpha+1}{\sigma}$ and $q=\infty$.

Remark 2.4. In the case of Theorem 2.3, we deduce from our proof that the sequence $\alpha+a j$ can be changed into $\alpha+a(j)$ where $\{a(j)\}_{j \geq 0}$, is a positive, increasing and unbounded sequence such that $a(0)=0$ and $a(1) \geq 1$. 


\section{An application of Theorem 2.3}

As we commented in the previous section, the results in Theorem 2.1 and Theorem 2.3 are extensions of some known inequalities for fractional integrals for Laguerre expansions. However, the inequality (2.3) appears in a natural way in the study of fractional integrals related to the harmonic oscillator. Indeed, the eigenfunctions of the harmonic oscillator in $\mathbb{R}^{n}$ verify

$$
\left(-\Delta+|\cdot|^{2}\right) \phi=E \phi
$$

where $E$ is the corresponding eigenvalue. There are two complete sets of eigenfunctions for this equation. Using cartesian coordinates, one obtains the functions

$$
\phi_{k}(x)=\prod_{i=1}^{n} h_{k_{i}}\left(x_{i}\right), \quad k=\left(k_{1}, \ldots, k_{n}\right) \in \mathbb{N}^{n},
$$

where $h_{k_{i}}\left(x_{i}\right)=\left(\sqrt{\pi} 2^{k_{i}} k_{i} !\right)^{-1 / 2} H_{k_{i}}\left(x_{i}\right) e^{-x_{i}^{2} / 2}$, and $H_{j}$ denote the Hermite polynomials of degree $j \in \mathbb{N}$ (see [17, p. 60]). In this case the eigenvalues are $E_{k}=2|k|+n$. The system of functions $\left\{\phi_{k}\right\}_{k \in \mathbb{N}^{n}}$ is orthonormal and complete in $L^{2}\left(\mathbb{R}^{n}, d x\right)$. The fractional integrals for this system have been studied in [4] and [20]. Vector-valued extensions of the results in both papers for sequences of functions $\left\{f_{j}(x)\right\}_{j \in \mathbb{N}}$, with $x \in \mathbb{R}^{n}$, are trivial.

But the situation is completely different if we analyze the eigenfunctions of the harmonic oscillator by using spherical coordinates. Let $\mathcal{H}_{j}$ be the space of spherical harmonics of degree $j$ in $n$ variables. Let $\left\{\mathcal{Y}_{j, \ell}\right\}_{\ell=1, \ldots, \operatorname{dim}} \mathcal{H}_{j}$ be an orthonormal basis for $\mathcal{H}_{j}$ in $L^{2}\left(\mathbb{S}^{n-1}, d \sigma\right)$. Then the eigenfunctions of the harmonic oscillator, see [10], are given by

$$
\tilde{\phi}_{m, j, \ell}(x)=\left(\frac{2 \Gamma(j+1)}{\Gamma(m-j+n / 2)}\right)^{1 / 2} L_{j}^{n / 2-1+m-2 j}\left(r^{2}\right) \mathcal{Y}_{m-2 j, \ell}(x) e^{-r^{2} / 2}, \quad r^{2}=x_{1}^{2}+\cdots+x_{n}^{2},
$$

where $m \geq 0, j=0, \ldots,\lfloor m / 2\rfloor, \ell=1, \ldots, \operatorname{dim} \mathcal{H}_{m-2 j}$, and $L_{j}^{b}$ are Laguerre polynomials of order $b$ and degree $j \in \mathbb{N}$. This system is orthonormal and complete in $L^{2}\left(\mathbb{R}^{n}, d x\right)$ and the associated eigenvalues are $E_{m, j, \ell}=(n+2 m)$. Moreover

$$
L^{2}\left(\mathbb{R}^{n}, d x\right)=\bigoplus_{m=0}^{\infty} \mathcal{J}_{m}
$$

with

$$
\mathcal{J}_{m}=\left\{f \in C^{\infty}\left(\mathbb{R}^{n}\right):\left(-\Delta+|\cdot|^{2}\right) f=(n+2 m) f\right\}
$$

For each $\sigma>0$, we define the fractional integrals for the harmonic oscillator as

$$
\left(-\Delta+|\cdot|^{2}\right)^{-\sigma} f=\sum_{m=0}^{\infty} \frac{1}{(n+2 m)^{\sigma}} \operatorname{Proj}_{\mathcal{J}_{m}} f
$$

where

$$
\operatorname{Proj}_{\mathcal{J}_{m}} f=\sum_{j=0}^{\left\lfloor\frac{m}{2}\right\rfloor} \sum_{\ell=1}^{\operatorname{dim} \mathcal{H}_{m-2 j}} c_{m, j, \ell}(f) \tilde{\phi}_{m, j, \ell}, \quad c_{m, j, \ell}(f)=\int_{\mathbb{R}^{n}} \overline{\tilde{\phi}_{m, j, \ell}}(y) f(y) d y .
$$

The most appropriate spaces in order to analyze this kind of operators are the mixed norm spaces, defined as

where

$$
L^{p, 2}\left(\mathbb{R}^{n}, r^{n-1} d r d \omega\right)=\left\{f(x):\|f\|_{L^{p, 2}\left(\mathbb{R}^{n}, r^{n-1} d r d \omega\right)}<\infty\right\}
$$

$$
\|f\|_{L^{p, 2}\left(\mathbb{R}^{n}, r^{n-1} d r d \omega\right)}=\left(\int_{0}^{\infty}\left(\int_{\mathbb{S}^{n-1}}\left|f\left(r x^{\prime}\right)\right|^{2} d \omega\left(x^{\prime}\right)\right)^{p / 2} r^{n-1} d r\right)^{1 / p}
$$

with the obvious modification in the case $p=\infty$. The main characteristic of these spaces is that we consider the $L^{2}$-norm in the angular part and the $L^{p}$-norm in the radial part. They are very different from $L^{p}\left(\mathbb{R}^{n}, d x\right)$; in fact $L^{p}\left(\mathbb{R}^{n}, d x\right) \subset L^{p, 2}\left(\mathbb{R}^{n}, r^{n-1} d r d \omega\right)$ for $p>2, L^{2}\left(\mathbb{R}^{n}, d x\right)=$ $L^{2,2}\left(\mathbb{R}^{n}, r^{n-1} d r d \omega\right)$, and $L^{p, 2}\left(\mathbb{R}^{n}, r^{n-1} d r d \omega\right) \subset L^{p}\left(\mathbb{R}^{n}, d x\right)$ for $p<2$. These spaces are suitable 
when spherical harmonics are involved. Indeed, if a function $f$ on $\mathbb{R}^{n}$ is expanded in spherical harmonics,

$$
f(x)=\sum_{j=0}^{\infty} \sum_{\ell=1}^{\operatorname{dim} \mathcal{H}_{j}} f_{j, \ell}(r) \mathcal{Y}_{j, \ell}\left(\frac{x}{r}\right)
$$

where

we have

$$
f_{j, \ell}(r)=\int_{\mathbb{S}^{d-1}} f\left(r x^{\prime}\right) \overline{\mathcal{Y}_{j, \ell}}\left(x^{\prime}\right) d \omega\left(x^{\prime}\right)
$$

$$
\|f\|_{L^{p, 2}\left(\mathbb{R}^{n}, r^{n-1} d r d \omega\right)}=\left\|\left(\sum_{j=0}^{\infty} \sum_{\ell=1}^{\operatorname{dim} \mathcal{H}_{j}}\left|f_{j, \ell}(r)\right|^{2}\right)^{1 / 2}\right\|_{L^{p}\left((0, \infty), d \mu_{n / 2-1}\right)} .
$$

From this and using Theorem 2.3, we can prove the following result.

Theorem 3.1. Let $n \geq 2,0<\sigma<n / 2$, and $1 \leq p, q \leq \infty$. Then there exists a constant depending only on $\sigma$ such that

$$
\left\|\left(-\Delta+|\cdot|^{2}\right)^{-\sigma} f\right\|_{L^{q, 2}\left(\mathbb{R}^{n}, r^{n-1} d r d \omega\right)} \leq C\|f\|_{L^{p, 2}\left(\mathbb{R}^{n}, r^{n-1} d r d \omega\right)}
$$

for all $f \in L^{p, 2}\left(\mathbb{R}^{n}, r^{n-1} d r d \omega\right)$, if and only if $p$ and $q$ satisfy

$$
\frac{1}{p}-\frac{2 \sigma}{n} \leq \frac{1}{q}<\frac{1}{p}+\frac{2 \sigma}{n},
$$

with exclusion of the cases $p=1$ and $q=\frac{n}{n-2 \sigma}$, and $p=\frac{n}{2 \sigma}$ and $q=\infty$.

Proof. Consider the decomposition

$$
\left(-\Delta+|\cdot|^{2}\right)^{-\sigma} f=O_{1} f+O_{2} f
$$

where

$$
O_{1} f=\sum_{k=0}^{\infty} \frac{1}{(n+4 k)^{\sigma}} \operatorname{Proj}_{\mathcal{J}_{2 k}} f \quad \text { and } \quad O_{2} f=\sum_{k=0}^{\infty} \frac{1}{(n+4 k+2)^{\sigma}} \operatorname{Proj}_{\mathcal{J}_{2 k+1}} f .
$$

We start analyzing $O_{1}$. After some elementary algebraic manipulations, we have

$$
O_{1} f=\sum_{j=0}^{\infty} \sum_{\ell=1}^{\operatorname{dim}} \sum_{k=0}^{\infty} \frac{1}{(n+4 j+4 k)^{\sigma}} c_{2 j+2 k, k, \ell}(f) \tilde{\phi}_{2 j+2 k, k, \ell} .
$$

By (3.1), we can deduce the following identity immediately

$$
c_{2 j+2 k, k, \ell}(f) \tilde{\phi}_{2 j+2 k, k, \ell}(x)=b_{k}^{n / 2-1+2 j}\left((\cdot)^{-2 j} f_{2 j, \ell}\right) r^{2 j} \psi_{k}^{n / 2-1+2 j}(r) \mathcal{Y}_{2 j, \ell}\left(\frac{x}{r}\right),
$$

where we used the notation in the previous section for Laguerre expansions of convolution type. Then

$$
O_{1} f(x)=\sum_{j=0}^{\infty} \sum_{\ell=1}^{\operatorname{dim} \mathcal{H}_{2 j}} r^{2 j}\left(L_{n / 2-1+2 j}\right)^{-\sigma}\left((\cdot)^{-2 j} f_{2 j, \ell}\right)(r) \mathcal{Y}_{2 j, \ell}\left(\frac{x}{r}\right) .
$$

In a similar way, we conclude that

$$
O_{2} f(x)=\sum_{j=0}^{\infty} \sum_{\ell=1}^{\operatorname{dim} \mathcal{H}_{2 j+1}} r^{2 j+1}\left(L_{n / 2+2 j}\right)^{-\sigma}\left((\cdot)^{-2 j-1} f_{2 j+1, \ell}\right)(r) \mathcal{Y}_{2 j+1, \ell}\left(\frac{x}{r}\right)
$$

and

$$
\left(-\Delta+|\cdot|^{2}\right)^{-\sigma} f(x)=\sum_{j=0}^{\infty} \sum_{\ell=1}^{\operatorname{dim} \mathcal{H}_{j}} r^{j}\left(L_{n / 2-1+j}\right)^{-\sigma}\left((\cdot)^{-j} f_{j, \ell}\right)(r) \mathcal{Y}_{j, \ell}\left(\frac{x}{r}\right) .
$$

So, the inequality $(3.2)$ is equivalent to 


$$
\begin{aligned}
\|\left(\sum_{j=0}^{\infty} \sum_{\ell=1}^{\operatorname{dim} \mathcal{H}_{j}}\left(r^{j}\left(L_{n / 2-1+j}\right)^{-\sigma}\left((\cdot)^{-j} f_{j, \ell}\right)(r)\right)^{2}\right)^{1 / 2} & \|_{L^{p}\left((0, \infty), d \mu_{n / 2-1}\right)} \\
& \leq C\left\|\left(\sum_{j=0}^{\infty} \sum_{\ell=1}^{\operatorname{dim} \mathcal{H}_{j}}\left(f_{j, \ell}(r)\right)^{2}\right)^{1 / 2}\right\|_{L^{p}\left((0, \infty), d \mu_{n / 2-1}\right)}
\end{aligned}
$$

Then the proof follows as a consequence of Theorem 2.3.

Remark 3.2. We have just proved that Theorem 3.1 is a consequence of the vector-valued estimates in Theorem 2.3. An alternative proof could be obtained by using the boundedness of the fractional integrals given in [4, Theorem 8] and an observation due to Rubio de Francia in [24, Remark (a)]. This has been pointed out to us by G. Garrigós in a personal communication.

\section{Proof of Theorem 2.1}

The heat semigroup related to $L_{\alpha}^{H}$ is initially defined in $L^{2}\left(\mathbb{R}_{+}^{n}, d x\right)$ as

$$
T_{\alpha, t}^{H} f=\sum_{m=0}^{\infty} e^{-t(4 m+2|\alpha|+2 n)} \sum_{|k|=m}\left\langle f, \varphi_{k}^{\alpha}\right\rangle \varphi_{k}^{\alpha}, \quad t>0,
$$

and by $\langle f, g\rangle$ we denote $\int_{\mathbb{R}_{+}^{n}} f(x) \overline{g(x)} d x$. We can write the heat semigroup $\left\{T_{\alpha, t}^{H}\right\}_{t>0}$ as an integral operator

$$
T_{\alpha, t}^{H} f(x)=\int_{\mathbb{R}_{+}^{n}} G_{\alpha, t}^{H}(x, y) f(y) d y
$$

where the Laguerre heat kernel is given by

$$
G_{\alpha, t}^{H}(x, y)=\sum_{m=0}^{\infty} e^{-t(4 m+2|\alpha|+2 n)} \sum_{|k|=m} \varphi_{k}^{\alpha}(x) \varphi_{k}^{\alpha}(y) .
$$

The explicit expression for the Laguerre heat kernel is known and it can be found in [17, (4.17.6)]:

$$
G_{\alpha, t}^{H}(x, y)=(\sinh 2 t)^{-n} \exp \left(-\frac{1}{2} \operatorname{coth}(2 t)\left(|x|^{2}+|y|^{2}\right)\right) \prod_{i=1}^{n}\left(x_{i} y_{i}\right)^{1 / 2} I_{\alpha_{i}}\left(\frac{x_{i} y_{i}}{\sinh 2 t}\right),
$$

with $I_{\nu}$ denoting the modified Bessel function of the first kind and order $\nu$, see [17, Chapter 5].

We use Schläfli's integral representation of Poisson's type for the modified Bessel function, see [17, $(5.10 .22)]$,

$$
I_{\nu}(z)=z^{\nu} \int_{-1}^{1} \exp (-z s) d \Pi_{\nu}(s), \quad|\arg z|<\pi, \quad \nu>-\frac{1}{2},
$$

where the measure $d \Pi_{\nu}(u)$ is given by

$$
d \Pi_{\nu}(u)=\frac{\left(1-u^{2}\right)^{\nu-1 / 2} d u}{\sqrt{\pi} 2^{\nu} \Gamma(\nu+1 / 2)}, \quad \nu>-1 / 2 .
$$

In the limit case $\nu=-1 / 2$, we put $\Pi_{-1 / 2}=\frac{1}{2}\left(\delta_{-1}+\delta_{1}\right)$. Consequently, for $\alpha \in[-1 / 2, \infty)^{n}$, the kernel can be expressed as

$G_{\alpha, t}^{H}(x, y)=(x y)^{\alpha+\mathbf{1} / 2}(\sinh (2 t))^{-n-|\alpha|} \int_{[-1,1]^{n}} \exp \left(-\frac{1}{2} \operatorname{coth}(2 t)\left(|x|^{2}+|y|^{2}\right)-\sum_{i=1}^{n} \frac{x_{i} y_{i} s_{i}}{\sinh (2 t)}\right) d \Pi_{\alpha}(s)$,

where $\mathbf{1}=(1, \ldots, 1) \in \mathbb{N}^{n}, x y=\left(x_{1} y_{1}, \ldots, x_{n} y_{n}\right), x^{\alpha}=x_{1}^{\alpha_{1}} \cdot \ldots \cdot x_{n}^{\alpha_{n}}$ and

$$
d \Pi_{\alpha}(s)=\prod_{i=1}^{n} \frac{\left(1-s_{i}^{2}\right)^{\alpha_{i}-1 / 2}}{2^{\alpha_{i}} \sqrt{\pi} \Gamma\left(\alpha_{i}+1 / 2\right)} d s_{i}
$$


Letting

$$
q_{ \pm}=q_{ \pm}(x, y, s)=|x|^{2}+|y|^{2} \pm 2 \sum_{i=1}^{n} x_{i} y_{i} s_{i}
$$

the change of variable

$$
t(\xi)=\frac{1}{2} \log \frac{1+\xi}{1-\xi}, \quad \xi \in(0,1)
$$

leads to

$$
G_{\alpha, t(\xi)}^{H}(x, y)=(x y)^{\alpha+\mathbf{1} / 2}\left(\frac{1-\xi^{2}}{2 \xi}\right)^{n+|\alpha|} \int_{[-1,1]^{n}} \exp \left(-\frac{1}{4 \xi} q_{+}(x, y, s)-\frac{\xi}{4} q_{-}(x, y, s)\right) d \Pi_{\alpha}(s) .
$$

The following technical lemma, which we will use to get estimates for the kernel $G_{\alpha, t(\xi)}^{H}$, can be found in [20, Lemma 2.1].

Lemma 4.1. Let $a \in \mathbb{R}$ be fixed and $T>0$. Then

$$
\int_{0}^{1} \zeta^{-a} \exp \left(-T \zeta^{-1}\right) d \zeta \leq C \exp (-T / 2), \quad T \geq 1
$$

and for $0<T<1$

$$
\int_{0}^{1} \zeta^{-a} \exp \left(-T \zeta^{-1}\right) d \zeta \simeq \begin{cases}T^{-a+1}, & a>1 \\ \log (2 / T), & a=1 \\ 1, & a<1\end{cases}
$$

Proposition 4.2. Let $\alpha \in[-1 / 2, \infty)^{n}$. Then

$$
G_{\alpha, t(\xi)}^{H}(x, y) \leq C\left(\frac{1-\xi^{2}}{\xi}\right)^{n / 2} \exp \left(-\frac{|x-y|^{2}}{4 \xi}-\frac{\xi|x+y|^{2}}{4}\right),
$$

with $C$ independent of $\alpha$.

Proof. Let $q_{ \pm, i}=q_{ \pm, i}\left(x_{i}, y_{i}, s_{i}\right)=x_{i}^{2}+y_{i}^{2} \pm 2 x_{i} y_{i} s_{i}$, for $i=1, \ldots, n$. From this identity and (4.2), it follows that $q_{ \pm}(x, y, s)=\sum_{i=1}^{n} q_{ \pm, i}\left(x_{i}, y_{i}, s_{i}\right)$. Observe that

$$
\int_{[-1,1]^{n}} \exp \left(-\frac{q_{+}}{4 \xi}-\frac{\xi q_{-}}{4}\right) d \Pi_{\alpha}(s)=\prod_{i=1}^{n} \int_{-1}^{1} \exp \left(-\frac{q_{+, i}}{4 \xi}-\frac{\xi q_{-, i}}{4}\right) d \Pi_{\alpha_{i}}\left(s_{i}\right),
$$

so it suffices to deal with the integral in dimension one. The case $\alpha_{i}=-1 / 2$ is elementary, so we obtain the estimate for $\alpha_{i}>-1 / 2$. We write

$$
J:=\int_{-1}^{1} \exp \left(-\frac{q_{+, i}}{4 \xi}-\frac{\xi q_{-, i}}{4}\right)\left(1-s_{i}^{2}\right)^{\alpha_{i}-1 / 2} d s_{i} .
$$

With the change of variable $s_{i}=2 u-1$ we have

$$
J=4^{\alpha_{i}} \exp \left(-\frac{\left(x_{i}-y_{i}\right)^{2}}{4 \xi}-\frac{\xi\left(x_{i}+y_{i}\right)^{2}}{4}\right) \int_{0}^{1} \exp \left(-x_{i} y_{i} u\left(\frac{1}{\xi}-\xi\right)\right) u^{\alpha_{i}-1 / 2}(1-u)^{\alpha_{i}-1 / 2} d u .
$$

It is easy to check that

$$
\begin{aligned}
\int_{1 / 2}^{1} \exp \left(-x_{i} y_{i} u\left(\frac{1}{\xi}-\xi\right)\right) u^{\alpha_{i}-1 / 2}(1 & -u)^{\alpha_{i}-1 / 2} d u \\
& \leq \int_{0}^{1 / 2} \exp \left(-x_{i} y_{i} u\left(\frac{1}{\xi}-\xi\right)\right) u^{\alpha_{i}-1 / 2}(1-u)^{\alpha_{i}-1 / 2} d u
\end{aligned}
$$

and then

$$
J \leq 4^{\alpha_{i}+1 / 2} \exp \left(-\frac{\left(x_{i}-y_{i}\right)^{2}}{4 \xi}-\frac{\xi\left(x_{i}+y_{i}\right)^{2}}{4}\right) \int_{0}^{1 / 2} \exp \left(-x_{i} y_{i} u\left(\frac{1}{\xi}-\xi\right)\right) u^{\alpha_{i}-1 / 2} d u .
$$


Now, taking $x_{i} y_{i} u\left(\frac{1}{\xi}-\xi\right)=z$, we get

$$
\begin{aligned}
J & \leq \frac{4^{\alpha_{i}+1 / 2}}{\left(x_{i} y_{i}\right)^{\alpha_{i}+1 / 2}}\left(\frac{\xi}{1-\xi^{2}}\right)^{\alpha_{i}+1 / 2} \exp \left(-\frac{\left(x_{i}-y_{i}\right)^{2}}{4 \xi}-\frac{\xi\left(x_{i}+y_{i}\right)^{2}}{4}\right) \int_{0}^{x_{i} y_{i}\left(1-\xi^{2}\right) /(2 \xi)} e^{-z} z^{\alpha_{i}-1 / 2} d z \\
& \leq \frac{4^{\alpha_{i}+1 / 2}}{\left(x_{i} y_{i}\right)^{\alpha_{i}+1 / 2}}\left(\frac{\xi}{1-\xi^{2}}\right)^{\alpha_{i}+1 / 2} \exp \left(-\frac{\left(x_{i}-y_{i}\right)^{2}}{4 \xi}-\frac{\xi\left(x_{i}+y_{i}\right)^{2}}{4}\right) \Gamma\left(\alpha_{i}+1 / 2\right) .
\end{aligned}
$$

From (4.1), (4.4) and (4.5), the estimate in the proposition follows.

For each $\sigma>0$, we define the potential operator

$$
\mathcal{I}_{\alpha, \sigma}^{H} f(x)=\int_{\mathbb{R}_{+}^{n}} \mathcal{H}_{\alpha, \sigma}^{H}(x, y) f(y) d y, \quad x \in \mathbb{R}_{+}^{n},
$$

where

$$
\mathcal{H}_{\alpha, \sigma}^{H}(x, y)=\frac{1}{\Gamma(\sigma)} \int_{0}^{\infty} G_{\alpha, t}^{H}(x, y) t^{\sigma-1} d t, \quad x, y \in \mathbb{R}_{+}^{n}
$$

is the potential kernel. On the other hand, following the notation in [20], we define the function $K_{\sigma}(x), x \in \mathbb{R}^{n} \backslash\{0\}$, by

$$
K_{\sigma}(x)=\exp \left(-c|x|^{2}\right), \quad|x| \geq 1, \quad K_{\sigma}(x)= \begin{cases}\frac{1}{|x|^{n-2 \sigma}}, & \sigma<n / 2, \\ \log \left(\frac{c}{|x|}\right), & \sigma=n / 2, \quad|x|<1 . \\ 1, & \sigma>n / 2,\end{cases}
$$

The estimate contained in the following proposition has sharper extensions in the existing literature, see [4, Proposition 2] and [28, Lemma 5.3]. In our case, we do not need so general estimates for our purposes, so we decided to include a short proof to make the paper self-contained.

Proposition 4.3. Let $\sigma>0$ and $\alpha \in[-1 / 2, \infty)^{n}$. Then

$$
\mathcal{H}_{\alpha, \sigma}^{H}(x, y) \leq C_{\sigma} K_{\sigma}(x-y)
$$

with $C_{\sigma}$ independent of $\alpha$.

Proof. By (4.6), the change of variable (4.3), (4.4), and Proposition 4.2, we have

$$
\begin{aligned}
\mathcal{H}_{\alpha, \sigma}^{H}(x, y) & \leq \frac{C}{2^{\sigma-1}} \int_{0}^{1}\left(\log \frac{1+\xi}{1-\xi}\right)^{\sigma-1}\left(1-\xi^{2}\right)^{n / 2-1} \xi^{-n / 2} \exp \left(-\frac{1}{4 \xi}|x-y|^{2}\right) d \xi \\
& =\int_{0}^{1 / 2}+\int_{1 / 2}^{1}=: I_{1}+I_{2} .
\end{aligned}
$$

Concerning $I_{1}$, observe that there exists $C$ such that $\log \frac{1+\xi}{1-\xi}<C \xi$, for $\xi \in(0,1 / 2)$. Then, we apply Lemma 4.1 with $a=-\sigma+1+n / 2$ and $T=|x-y|^{2} / 4$, and we obtain

$$
I_{1} \leq C_{\sigma} \int_{0}^{1 / 2} \xi^{\sigma-1-n / 2} \exp \left(-\frac{1}{4 \xi}|x-y|^{2}\right) d \xi \leq C_{\sigma} \begin{cases}\frac{1}{|x-y|^{n-2 \sigma}}, & \sigma<n / 2, \\ \log \left(\frac{c}{|x-y|}\right), & \sigma=n / 2 \\ 1, & \sigma>n / 2\end{cases}
$$

for $|x-y|<2$, and

$$
I_{1} \leq C_{\sigma} \exp \left(-c|x-y|^{2}\right)
$$

for $|x-y| \geq 2$, which is equivalent to the required bound. Now we deal with $I_{2}$. By using that $\xi^{-n / 2} \sim 1$, for $\xi \in(1 / 2,1)$, and reverting the change of variable (4.3) yield

$$
\begin{aligned}
I_{2} & \leq C_{\sigma} \exp \left(-c|x-y|^{2}\right) \int_{1 / 2}^{1}\left(\log \left(\frac{1+\xi}{1-\xi}\right)\right)^{\sigma-1}\left(1-\xi^{2}\right)^{n / 2-1} d \xi \\
& \leq C_{\sigma} \exp \left(-c|x-y|^{2}\right) \int_{\log 3}^{\infty} w^{\sigma-1} \exp (-w n / 2) d w \leq C_{\sigma} \exp \left(-c|x-y|^{2}\right),
\end{aligned}
$$


which is enough for our purposes.

Remark 4.4. We emphasize that, although the previous proposition may be known in the literature as a technical lemma from the point of view of fractional integrals for the harmonic oscillator, it is new in the sense that we are estimating the kernel of the fractional integrals for the Laguerre operator with a constant independent of the parameter $\alpha$. Of course, the important estimate employed to get Proposition 4.3 is the one proved in Proposition 4.2, which delivers an estimate for the heat kernel with a constant independent of $\alpha$.

Moreover, to complete the proof of Theorem 2.1, we will use the following result about vectorvalued extensions of bounded operators. This result is a version of [14, Ch. 5, Theorem 1.12] in the setting of $\ell^{r}$ spaces.

Lemma 4.5. Consider $L^{p}=L^{p}(X, m)$, where $(X, m)$ is a measure space. Let $T: L^{p} \rightarrow L^{q}$ be a bounded linear operator which is positive (i.e. $g(x) \geq 0$ implies $T g(x) \geq 0$ ), $1 \leq p, q \leq \infty$, with norm $\|T\|$. Then $T$ has an $\ell^{r}$-valued extension for $1 \leq r \leq \infty$ and

$$
\left\|\left(\sum_{j}\left|T f_{j}\right|^{r}\right)^{1 / r}\right\|_{L^{q}} \leq\|T\|\left\|\left(\sum_{j}\left|f_{j}\right|^{r}\right)^{1 / r}\right\|_{L^{p}}, \quad f_{j} \in L^{p} .
$$

Proof of Theorem 2.1. First of all, analogously as in [20], we shall show that $\left(L_{\alpha}^{H}\right)^{-\sigma}=\mathcal{I}_{\alpha, \sigma}^{H}$ on $L^{2}\left(\mathbb{R}_{+}^{n}, d x\right)$. Then, it suffices to prove that $\left(L_{\alpha}^{H}\right)^{-\sigma} \varphi_{k}^{\alpha}=\mathcal{I}_{\alpha, \sigma}^{H} \varphi_{k}^{\alpha}$. We have

$$
\begin{aligned}
\int_{\mathbb{R}_{+}^{n}} \mathcal{H}_{\alpha, \sigma}^{H}(x, y) \varphi_{k}^{\alpha}(y) d y & =\int_{\mathbb{R}_{+}^{n}} \int_{0}^{\infty} G_{\alpha, t}^{H}(x, y) t^{\sigma-1} d t \varphi_{k}^{\alpha}(y) d y \\
& =\varphi_{k}^{\alpha}(x) \int_{0}^{\infty} e^{-t(4|k|+2|\alpha|+2 n)} t^{\sigma-1} d t=\Gamma(\sigma)\left(L_{\alpha}^{H}\right)^{-\sigma} \varphi_{k}^{\alpha}(x)
\end{aligned}
$$

and the application of Fubini's theorem in the second identity is justified because $\mathcal{H}_{\alpha, \sigma}^{H}(x, \cdot) \leq K_{\sigma}(x-$ $\cdot) \in L^{1}\left(\mathbb{R}^{n}, d x\right)$, for each $x \in \mathbb{R}_{+}^{n}$, and $\varphi_{k}^{\alpha} \in L^{\infty}\left(\mathbb{R}_{+}^{n}, d x\right)$.

Now we observe that, by Proposition 4.3, there exists a constant $C_{\sigma}$ depending only on $\sigma$ such that for a nonnegative function $f$,

$$
\mathcal{I}_{\alpha+a j, \sigma}^{H}(f)(x) \leq C_{\sigma} \int_{\mathbb{R}_{+}^{n}} K_{\sigma}(x-y) f(y) d y .
$$

By [20, Theorem 2.5] and Lemma 4.5 (note that $K_{\sigma}(x-y)$ is positive), there exists a constant $C$ depending only on $\sigma, s$ and $t$ such that

$$
\left\|\left(\sum_{j=0}^{\infty}\left|\int_{\mathbb{R}_{+}^{n}} K_{\sigma}(x-y) f_{j}(y) d y\right|^{r}\right)^{1 / r}\right\|\left\|_{L^{q}\left(\mathbb{R}_{+}^{n},|x|^{-s q} d x\right)} \leq C\right\|\left(\sum_{j=0}^{\infty}\left|f_{j}\right|^{r}\right)^{1 / r} \|_{L^{p}\left(\mathbb{R}_{+}^{n},|x|^{t p} d x\right)^{\prime}},
$$

for $f_{j} \in L^{p}\left(\mathbb{R}_{+}^{n},|x|^{t p} d x\right)$.

Therefore,

$$
\left\|\left(\sum_{j=0}^{\infty}\left|\mathcal{I}_{\alpha+a j, \sigma}^{H}\left(f_{j}\right)\right|^{r}\right)^{1 / r}\right\|_{L^{q}\left(\mathbb{R}_{+}^{n},|x|^{-s q} d x\right)} \leq C\left\|\left(\sum_{j=0}^{\infty}\left|f_{j}\right|^{r}\right)^{1 / r}\right\|_{L^{p}\left(\mathbb{R}_{+}^{n},|x|^{t p} d x\right)},
$$

and the proof is complete.

\section{Proof of Theorem 2.3}

Recall the differential operator $L_{\alpha}$ given in (2.2). The heat semigroup related to $L_{\alpha}$ is initially defined in $L^{2}\left((0, \infty), d \mu_{\alpha}\right)$ as

$$
T_{\alpha, t} f=\sum_{m=0}^{\infty} e^{-t(4 m+2 \alpha+2)}\left\langle f, \psi_{m}^{\alpha}\right\rangle_{d \mu_{\alpha}} \psi_{m}^{\alpha}, \quad t>0
$$


and by $\langle f, g\rangle_{d \mu_{\alpha}}$ we denote $\int_{0}^{\infty} f(r) \overline{g(r)} d \mu_{\alpha}(r)$. We can write the heat semigroup $\left\{T_{\alpha, t}\right\}_{t>0}$ as an integral operator

$$
T_{\alpha, t} f(r)=\int_{0}^{\infty} G_{\alpha, t}(r, s) f(s) d \mu_{\alpha}(s),
$$

where the Laguerre heat kernel in this case is given by

$$
G_{\alpha, t}(r, s)=\sum_{m=0}^{\infty} e^{-t(4 m+2 \alpha+2)} \psi_{m}^{\alpha}(r) \psi_{m}^{\alpha}(s) .
$$

Observe that

$$
G_{\alpha, t}(r, s)=G_{\alpha, t}^{H}(r, s)(r s)^{-\alpha-1 / 2},
$$

with $G_{\alpha, t}^{H}$ considered now as a one-dimensional kernel.

We define the potential operator

$$
\mathcal{I}_{\alpha, \sigma} f(r)=\int_{0}^{\infty} \mathcal{H}_{\alpha, \sigma}(r, s) f(s) d \mu_{\alpha}(s), \quad r>0,
$$

where

$$
\mathcal{H}_{\alpha, \sigma}(r, s)=\frac{1}{\Gamma(\sigma)} \int_{0}^{\infty} G_{\alpha, t}(r, s) t^{\sigma-1} d t, \quad r, s>0,
$$

is the potential kernel. Due to (5.1),

$$
\mathcal{H}_{\alpha, \sigma}(r, s)=\mathcal{H}_{\alpha, \sigma}^{H}(r, s)(r s)^{-\alpha-1 / 2},
$$

where $\mathcal{H}_{\alpha, \sigma}^{H}$ is the kernel in (4.6) considered in one-dimension. By using the estimates in the previous section for $\mathcal{H}_{\alpha, \sigma}^{H}$ and the relation (5.4), we obtain that

$$
\mathcal{H}_{\alpha+a j, \sigma}(r, s) \leq C_{\sigma} K_{\sigma}(r-s)(r s)^{-\alpha-a j-1 / 2} .
$$

If one uses this estimate to attain $L^{p}-L^{q}$ inequalities, then it turns out that we need an extra restriction on the parameters. In fact, the condition $\frac{4(\alpha+1)}{2 \alpha+3}<p \leq q<\frac{4(\alpha+1)}{2 \alpha+1}$ arises in the boundedness of $\mathcal{I}_{\alpha+a j, \sigma}$ due to the presence of the factor $(r s)^{-\alpha-1 / 2}$ and the measure $d \mu_{\alpha}$. So, in order to eliminate such restriction on $p$ and $q$, one has to get suitable estimates for the kernel. In this way, the proof of Theorem 2.3 is based on the estimates collected in Propositions 5.1 and 5.6.

Proposition 5.1. Let $\alpha \geq-1 / 2, a \geq 1, j$ be an integer number such that $j \geq 1$, and $0<\sigma<\alpha+1$. Then

$$
\mathcal{H}_{\alpha+a j, \sigma}(r, s) \leq C(r s)^{-a j} \mathcal{K}_{\alpha, \sigma}(r, s), \quad r, s \in(0, \infty),
$$

where $C$ depends on $\alpha$ and $\sigma$, but not on $j$, and

$$
\mathcal{K}_{\alpha, \sigma}(r, s)=\frac{1}{(r+s)^{2 \alpha+1}} \begin{cases}W_{\alpha, \sigma}(r, s), & |r-s|<1, \\ e^{-c(r-s)^{2}}, & |r-s| \geq 1,\end{cases}
$$

for some constant $c>0$, with

$$
W_{\alpha, \sigma}(r, s)= \begin{cases}|r-s|^{2 \sigma-1}, & \sigma<1 / 2, \\ \log \left(\frac{r+s}{|r-s|}\right), & \sigma=1 / 2, \\ \min \left\{(r+s)^{2 \sigma-1}, 1\right\}, & \sigma>1 / 2 .\end{cases}
$$

The two following lemmas will provide us the main tools to prove the previous proposition.

Lemma 5.2. Let $c>-1$ and $\ell$ be such that $0<\sigma<c+\ell$ and $a>0$. Then

$$
\int_{0}^{1}\left(\log \left(\frac{1+\xi}{1-\xi}\right)\right)^{\sigma-1}\left(1-\xi^{2}\right)^{c} \xi^{-c-\ell} \exp \left(-\frac{a}{4 \xi}\right) d \xi \leq C \frac{4^{c} \Gamma(c+\ell-\sigma)}{a^{c+\ell-\sigma}},
$$

where $C$ is independent of $c$. 
Lemma 5.3. Let $\alpha \geq-1 / 2, \lambda \in \mathbb{R}, b \geq 1,0<B<A$, and

$$
I_{\alpha, b}^{\lambda}=\int_{0}^{1} \frac{(1-s)^{\alpha+b-1 / 2}}{(A-B s)^{\alpha+b+\lambda+1 / 2}} d s .
$$

Then, for $\lambda>0$,

$$
I_{\alpha, b}^{\lambda} \leq \frac{\Gamma(b) \Gamma(\lambda)}{\Gamma(b+\lambda)} \frac{1}{A^{\alpha+1 / 2}} \frac{1}{B^{b}} \frac{1}{(A-B)^{\lambda}}
$$

for $\lambda=0$,

and, for $\lambda<0$,

$$
I_{\alpha, b}^{0} \leq \frac{1}{A^{\alpha+1 / 2}} \frac{1}{B^{b}} \log \left(\frac{A}{A-B}\right)
$$

$$
I_{\alpha, b}^{\lambda} \leq C \frac{1}{A^{\alpha+\lambda+1 / 2}} \frac{1}{B^{b}} .
$$

Additionally, for the case $|r-s|<1, r+s>2$, and $\sigma>1 / 2$ we will use the following result, which is an immediate consequence of (5.1) and Proposition 4.2.

Lemma 5.4. Let $\alpha \geq-1 / 2, a \geq 1$, and $j \in \mathbb{N}$. Then

$$
G_{\alpha+a j, t(\xi)}(r, s) \leq C \frac{\left(1-\xi^{2}\right)^{1 / 2}}{\xi^{1 / 2}}(r s)^{-a j-\alpha-1 / 2}, \quad r, s \in(0, \infty),
$$

with $C$ a constant independent of $j$.

Also, in the case $|r-s| \geq 1$, the result will follow from an appropriate estimate of the heat kernel.

Lemma 5.5. Let $\alpha \geq-1 / 2, a \geq 1$, and $j$ be an integer number such that $j \geq 1$. Then

$$
\begin{array}{r}
G_{\alpha+a j, t(\xi)}(r, s) \leq C \frac{\Gamma(a j)}{\Gamma(\alpha+a j+1 / 2)} \exp \left(-\frac{(r-s)^{2}}{8 \xi}\right) \frac{\left(1-\xi^{2}\right)^{\alpha+1}}{\xi^{\alpha+1}}\left|r^{2}-s^{2}\right|^{-(2 \alpha+1)}(r s)^{-a j}, \\
r, s \in(0, \infty),
\end{array}
$$

with $C$ a constant independent of $j$.

Lemmas 5.2, 5.3 and 5.5 are rather technical and their proof will be given in the last section.

Proof of Proposition 5.1. For $|r-s|<1$, by (5.3), (5.1), the change of variable (4.3), (4.4), Lemma 5.2 with $c=\alpha+a j, \ell=1$ and $a=q_{+}$, and the change of variable $v=1-2 u$, we have

$$
\begin{aligned}
\mathcal{H}_{\alpha+a j, \sigma}(r, s)= & C \int_{0}^{\infty} G_{\alpha+a j, t}^{H}(r, s)(r s)^{-\alpha-a j-1 / 2} t^{\sigma-1} d t \\
= & C \int_{0}^{1}\left(1-\xi^{2}\right)^{\alpha+a j}(2 \xi)^{-1-\alpha-a j}\left(\log \left(\frac{1+\xi}{1-\xi}\right)\right)^{\sigma-1} \\
& \times\left(\int_{-1}^{1} \exp \left(-\frac{1}{4 \xi} q_{+}(r, s, v)-\frac{\xi}{4} q_{-}(r, s, v)\right) d \Pi_{\alpha+a j}(v)\right) d \xi \\
\leq & C \frac{\Gamma(\alpha+a j+1-\sigma)}{\Gamma(\alpha+a j+1 / 2)} \int_{-1}^{1} \frac{\left(1-v^{2}\right)^{\alpha+a j-1 / 2}}{\left(q_{+}(r, s, v)\right)^{\alpha+a j+1-\sigma}} d v \\
= & C 4^{\alpha+a j} \frac{\Gamma(\alpha+a j+1-\sigma)}{\Gamma(\alpha+a j+1 / 2)} \int_{0}^{1} \frac{(1-u)^{\alpha+a j-1 / 2} u^{\alpha+a j-1 / 2}}{\left((r+s)^{2}-4 r s u\right)^{\alpha+a j+1-\sigma}} d u \\
\leq & C 4^{\alpha+a j} \frac{\Gamma(\alpha+a j+1-\sigma)}{\Gamma(\alpha+a j+1 / 2)} \int_{0}^{1} \frac{(1-u)^{\alpha+a j-1 / 2}}{\left((r+s)^{2}-4 r s u\right)^{\alpha+a j+1-\sigma}} d u .
\end{aligned}
$$

We conclude by using Lemma 5.3 with $b=a j, \lambda=1 / 2-\sigma, A=(r+s)^{2}$, and $B=4 r s$. Indeed, for $\sigma<1 / 2$,

$$
\mathcal{H}_{\alpha+a j, \sigma}(r, s) \leq C 4^{\alpha+a j} \frac{\Gamma(\alpha+a j+1-\sigma)}{\Gamma(\alpha+a j+1 / 2)} \frac{\Gamma(a j)}{\Gamma(a j+1 / 2-\sigma)} \frac{1}{(r+s)^{2 \alpha+1}} \frac{1}{(4 r s)^{a j}} \frac{1}{|r-s|^{1-2 \sigma}}
$$




$$
\leq C \frac{1}{(r+s)^{2 \alpha+1}} \frac{1}{(r s)^{a j}} \frac{1}{|r-s|^{1-2 \sigma}}
$$

for $\sigma=1 / 2$

$$
\mathcal{H}_{\alpha+a j, \sigma}(r, s) \leq C \frac{1}{(r+s)^{2 \alpha+1}} \frac{1}{(r s)^{a j}} \log \left(\frac{r+s}{|r-s|}\right)
$$

and, for $\sigma>1 / 2$,

$$
\mathcal{H}_{\alpha+a j, \sigma}(r, s) \leq C \frac{1}{(r+s)^{2 \alpha+2-2 \sigma}} \frac{1}{(r s)^{a j}} .
$$

The previous estimate for $\mathcal{H}_{\alpha+a j, \sigma}$, in the case $\sigma>1 / 2$, will be used when $\max \{r, s\} \leq 2$ and $|r-s|<1$. For $\max \{r, s\}>2$ and $|r-s|<1$, we obtain a sharper inequality by using Lemma 5.4. Indeed,

$$
\begin{aligned}
\mathcal{H}_{\alpha+a j, \sigma}(r, s) & \leq C(r s)^{-a j-\alpha-1 / 2} \int_{0}^{1}\left(\log \left(\frac{1+\xi}{1-\xi}\right)\right)^{\sigma-1}\left(1-\xi^{2}\right)^{-1 / 2} \xi^{-1 / 2} d \xi \\
& \leq C(r s)^{-a j}(r+s)^{-(2 \alpha+1)}
\end{aligned}
$$

where we have used that in this region $r s \sim(r+s)^{2}$ and that the integral is convergent because $\sigma>1 / 2$.

In order to bound the kernel in the case $|r-s| \geq 1$, we use (5.3), the change of variable (4.3), and Lemma 5.5 to obtain

$$
\begin{aligned}
\mathcal{H}_{\alpha+a j, \sigma}(r, s) \leq C(r & +s)^{-(2 \alpha+1)}(r s)^{-a j} \exp \left(-\frac{(r-s)^{2}}{16}\right) \\
& \times \int_{0}^{1}\left(\log \left(\frac{1+\xi}{1-\xi}\right)\right)^{\sigma-1}\left(1-\xi^{2}\right)^{\alpha} \xi^{-\alpha-1} \exp \left(-\frac{(r-s)^{2}}{16 \xi}\right) d \xi
\end{aligned}
$$

The last integral can be controlled by a constant after applying Lemma 5.2 with $c=\alpha$ and $\ell=1$, and the condition $|r-s| \geq 1$. Then

$$
\mathcal{H}_{\alpha+a j, \sigma}(r, s) \leq C(r+s)^{-(2 \alpha+1)}(r s)^{-a j} \exp \left(-\frac{(r-s)^{2}}{16}\right)
$$

and the proof is finished.

The next auxiliary result will be used in the proof of Theorem 2.3. The proof is also contained in the last section.

Proposition 5.6. Let $\alpha \geq-1 / 2, a \geq 1, j \in \mathbb{N}$, and $0<\sigma<\alpha+1$. Then, for all $r, s>0$,

$$
u_{j}(r)\left(L_{\alpha+a j}\right)^{-\sigma}\left(u_{j}^{-1}(\cdot) f\right)(r) \leq C \int_{0}^{\infty} f(s) \overline{\mathcal{K}}_{\alpha, \sigma}(r, s) d \mu_{\alpha}(s),
$$

where $C$ is independent of $j$ and

$$
\overline{\mathcal{K}}_{\alpha, \sigma}(r, s)=(r s)^{-\alpha-1 / 2} \int_{0}^{1}\left(\log \left(\frac{1+\xi}{1-\xi}\right)\right)^{\sigma-1} \xi^{-1 / 2}\left(1-\xi^{2}\right)^{-1 / 2} \exp \left(-\frac{(r-s)^{2}}{4 \xi}-\frac{\xi(r+s)^{2}}{4}\right) d \xi
$$

Moreover, for $r>2$,

$$
r^{2 \sigma} \int_{0}^{\infty} \overline{\mathcal{K}}_{\alpha, \sigma}(r, s) d \mu_{\alpha}(s) \leq C
$$

The proof of the necessity of the condition (2.4) in Theorem 2.3 is an immediate consequence of Theorem 2.2 in $[22]$ where the boundedness of $\left(L_{\alpha}\right)^{-\sigma}$ is characterized. The sufficiency of $(2.4)$ will be obtained from the following result and the Riesz-Thorin interpolation theorem.

Theorem 5.7. Let $\alpha \geq-1 / 2,0<\sigma<\alpha+1, a \geq 1$, and $1 \leq p, q, s \leq \infty$. Then: 
a) If $1-\frac{\sigma}{\alpha+1}<\frac{1}{q} \leq 1$, there exists a constant $C$ such that

$$
\left\|\left(\sum_{j=0}^{\infty}\left|u_{j}\left(L_{\alpha+a j}\right)^{-\sigma}\left(u_{j}^{-1} f_{j}\right)\right|^{s}\right)^{1 / s}\right\|_{L^{q}\left((0, \infty), d \mu_{\alpha}\right)} \leq C\left\|\left(\sum_{j=0}^{\infty}\left|f_{j}\right|^{s}\right)^{1 / s}\right\|_{L^{1}\left((0, \infty), d \mu_{\alpha}\right)} .
$$

b) If $\frac{1}{p}<\frac{\sigma}{\alpha+1}$, there exists a constant $C$ such that

$$
\left\|\left(\sum_{j=0}^{\infty}\left|u_{j}\left(L_{\alpha+a j}\right)^{-\sigma}\left(u_{j}^{-1} f_{j}\right)\right|^{s}\right)^{1 / s}\right\|_{L^{\infty}\left((0, \infty), d \mu_{\alpha}\right)} \leq C\left\|\left(\sum_{j=0}^{\infty}\left|f_{j}\right|^{s}\right)^{1 / s}\right\|_{L^{p}\left((0, \infty), d \mu_{\alpha}\right)} .
$$

c) If $\frac{1}{q}<\frac{\sigma}{\alpha+1}$, there exists a constant $C$ such that

$$
\left\|\left(\sum_{j=0}^{\infty}\left|u_{j}\left(L_{\alpha+a j}\right)^{-\sigma}\left(u_{j}^{-1} f_{j}\right)\right|^{s}\right)^{1 / s}\right\|_{L^{q}\left((0, \infty), d \mu_{\alpha}\right)} \leq C\left\|\left(\sum_{j=0}^{\infty}\left|f_{j}\right|^{s}\right)^{1 / s}\right\|_{L^{\infty}\left((0, \infty), d \mu_{\alpha}\right)} .
$$

d) If $1-\frac{\sigma}{\alpha+1}<\frac{1}{p} \leq 1$, there exists a constant $C$ such that

$$
\left\|\left(\sum_{j=0}^{\infty}\left|u_{j}\left(L_{\alpha+a j}\right)^{-\sigma}\left(u_{j}^{-1} f_{j}\right)\right|^{s}\right)^{1 / s}\right\|_{L^{1}\left((0, \infty), d \mu_{\alpha}\right)} \leq C\left\|\left(\sum_{j=0}^{\infty}\left|f_{j}\right|^{s}\right)^{1 / s}\right\|_{L^{p}\left((0, \infty), d \mu_{\alpha}\right)} .
$$

e) If $p>1, q<\infty$ and $\frac{1}{p}-\frac{\sigma}{\alpha+1}=\frac{1}{q}$, there exists a constant $C$ such that

$$
\left\|\left(\sum_{j=0}^{\infty}\left|u_{j}\left(L_{\alpha+a j}\right)^{-\sigma}\left(u_{j}^{-1} f_{j}\right)\right|^{s}\right)^{1 / s}\right\|_{L^{q}\left((0, \infty), d \mu_{\alpha}\right)} \leq C\left\|\left(\sum_{j=0}^{\infty}\left|f_{j}\right|^{s}\right)^{1 / s}\right\|_{L^{p}\left((0, \infty), d \mu_{\alpha}\right)} .
$$

The proof of e) is a consequence of a classical result for the Hardy operator (see [13, Theorem $1]$ ) and another result about the boundedness of local one-dimensional fractional integrals (see [12, Corollary 5.4]). These results are stated below.

Theorem 5.8. Let $f$ be a nonnegative function defined on $(0, \infty)$. Define $F(r)=\int_{0}^{r} f(t) d t$, if $\gamma>-1$, and $F(r)=\int_{r}^{\infty} f(t) d t$, if $\gamma<-1$. For $1 \leq p \leq q, \gamma \neq-1$, we have

$$
\left(\int_{0}^{\infty} F(r)^{q} r^{-1-q(\gamma+1)} d r\right)^{1 / q} \leq B(p, q, \gamma)\left(\int_{0}^{\infty} f(r)^{p} r^{-1-p \gamma} d r\right)^{1 / p}
$$

Theorem 5.9. Let $f$ be a nonnegative function defined on $(0, \infty)$. Let $0<\beta<1$ and consider the local fractional integral in $(0, \infty)$ :

$$
L_{\beta}^{\text {loc }} f(r)=\int_{r / 2}^{3 r / 2} \frac{f(t)}{|r-t|^{1-\beta}} d t
$$

Then

$$
\left(\int_{0}^{\infty} L_{\beta}^{\mathrm{loc}} f(r)^{q} r^{a} d r\right)^{1 / q} \leq C\left(\int_{0}^{\infty} f(r)^{p} r^{b} d r\right)^{1 / p}
$$

holds for $1 \leq p \leq q<\infty$ if and only if $(b+1) / p-(a+1) / q=\beta$ and either $1 / p-1 / q \leq \beta$ for $p>1$, or $1 / p-1 / q<\beta$ for $p=1$.

Proof of Theorem 5.7. As in the proof of Theorem 2.1, it can be checked easily that $\left(L_{\alpha}\right)^{-\sigma}=\mathcal{I}_{\alpha, \sigma}$ so we omit the details.

In the five parts of the proof we will analyze $u_{j}\left(L_{\alpha+a j}\right)^{-\sigma}\left(u_{j}^{-1} f_{j}\right)$ for $j \geq 1$. The case $j=0$ can be deduced from Theorem 2.2 in [22].

By (5.2) and Proposition 5.1, it is clear that

$$
u_{j}(r)\left(L_{\alpha+a j}\right)^{-\sigma}\left(u_{j}^{-1}(\cdot) f_{j}\right)(r) \leq C \int_{0}^{\infty} f_{j}(s) \mathcal{K}_{\alpha, \sigma}(r, s) d \mu_{\alpha}(s),
$$

where $\mathcal{K}_{\alpha, \sigma}$ is that in $(5.5)$. 
Proof of a) The inequality in a) will be deduced from the estimate

$$
\left\|\int_{0}^{\infty} f(s) \mathcal{K}_{\alpha, \sigma}(r, s) d \mu_{\alpha}(s)\right\|_{L^{q}\left((0, \infty), d \mu_{\alpha}\right)} \leq C\|f\|_{L^{1}\left((0, \infty), d \mu_{\alpha}\right)}
$$

and Lemma 4.5. Applying Minkowski's inequality, the previous inequality is a consequence of the estimate

$$
\left\|\mathcal{K}_{\alpha, \sigma}(\cdot, s)\right\|_{L^{q}\left((0, \infty), d \mu_{\alpha}\right)} \leq C, \quad 1 \leq q<\infty
$$

To prove the previous bound, we consider the cases $|r-s|<1$ and $|r-s| \geq 1$. For $|r-s|<1$ we distinguish three cases:

Case $\sigma<1 / 2$. For $s \geq 1$, we have

$$
\int_{|r-s|<1}\left(\mathcal{K}_{\alpha, \sigma}(r, s)\right)^{q} d \mu_{\alpha}(r) \leq C s^{(2 \alpha+2)(1-q)+2 q \sigma} \int_{|t-1|<1 / s}|1-t|^{-q(1-2 \sigma)} d t \sim s^{(2 \alpha+1)(1-q)} \leq C .
$$

When $s<1$, it is verified that

$$
\begin{aligned}
\int_{|r-s|<1}\left(\mathcal{K}_{\alpha, \sigma}(r, s)\right)^{q} d \mu_{\alpha}(r) & \leq C s^{(2 \alpha+2)(1-q)+2 q \sigma} \int_{0}^{1+1 / s} \frac{t^{2 \alpha+1}}{|1-t|^{q(1-2 \sigma)}(1+t)^{q(2 \alpha+1)}} d t \\
& \sim s^{(2 \alpha+2)(1-q)+2 q \sigma}\left(\int_{0}^{2}|1-t|^{-q(1-2 \sigma)} d t+\int_{2}^{1+1 / s} t^{2 \alpha+1-q(2 \alpha+2-2 \sigma)} d t\right) \\
& \sim s^{(2 \alpha+2)(1-q)+2 q \sigma}+1 \leq C,
\end{aligned}
$$

where in the last step we used that $1-\frac{\sigma}{\alpha+1}<\frac{1}{q}$.

Case $\sigma=1 / 2$. Observe that in this case $\alpha$ cannot be $-1 / 2$, due to the general assumptions in the theorem involving $\sigma$ and $\alpha$. For $s \geq 1$, we have

$$
\int_{|r-s|<1}\left(\mathcal{K}_{\alpha, \sigma}(r, s)\right)^{q} d \mu_{\alpha}(r) \leq C s^{(2 \alpha+2)(1-q)+q} \int_{|t-1|<1 / s}\left[\log \left(\frac{t+1}{|t-1|}\right)\right]^{q} d t .
$$

And the last integral can be controlled as follows

$$
\int_{|t-1|<1 / s}\left[\log \left(\frac{t+1}{|t-1|}\right)\right]^{q} d t \sim \int_{|t-1|<1 / s}(-\log |t-1|)^{q} d t \sim \int_{\log s}^{\infty} u^{q} e^{-u} d u \leq C .
$$

For $s<1$, it holds that

$$
\begin{aligned}
\int_{|r-s|<1}\left(\mathcal{K}_{\alpha, \sigma}(r, s)\right)^{q} d \mu_{\alpha}(r) & \leq C s^{(2 \alpha+2)(1-q)+q} \int_{0}^{1+1 / s} \frac{t^{2 \alpha+1}}{(1+t)^{q(2 \alpha+1)}}\left[\log \left(\frac{t+1}{|t-1|}\right)\right]^{q} d t \\
& \sim s^{(2 \alpha+2)(1-q)+q}+1 \leq C,
\end{aligned}
$$

where the condition $1-\frac{1}{2 \alpha+2}<\frac{1}{q}$ is used.

Case $\sigma>1 / 2$. For $s \geq 1$, we have

$$
\int_{|r-s|<1}\left(\mathcal{K}_{\alpha, \sigma}(r, s)\right)^{q} d \mu_{\alpha}(r) \leq C s^{(2 \alpha+2)(1-q)+q} \int_{|t-1|<1 / s} d t \sim s^{(2 \alpha+1)(1-q)} \leq C .
$$

When $s<1$, it is verified that

$$
\begin{aligned}
\int_{|r-s|<1}\left(\mathcal{K}_{\alpha, \sigma}(r, s)\right)^{q} d \mu_{\alpha}(r) & \leq C s^{(2 \alpha+2)(1-q)+2 q \sigma} \int_{0}^{1+1 / s} \frac{t^{2 \alpha+1}}{(1+t)^{q(2 \alpha+2-2 \sigma)}} d t \\
& \sim s^{(2 \alpha+2)(1-q)+2 q \sigma}+1 \leq C,
\end{aligned}
$$

where we use again the condition $1-\frac{\sigma}{\alpha+1}<\frac{1}{q}$. 
We pass to analyze (5.8) for $|r-s| \geq 1$. We have

$$
\int_{|r-s| \geq 1}\left(\mathcal{K}_{\alpha, \sigma}(r, s)\right)^{q} d \mu_{\alpha}(r) \leq C \int_{|r-s| \geq 1} \frac{e^{-c(r-s)^{2}}}{(r+s)^{q(2 \alpha+1)}} d \mu_{\alpha}(r) .
$$

In this region, the inequality $r^{2 \alpha+1}(r+s)^{-q(2 \alpha+1)} \leq C$ holds, then

$$
\int_{|r-s| \geq 1}\left(\mathcal{K}_{\alpha, \sigma}(r, s)\right)^{q} d \mu_{\alpha}(r) \leq C \int_{|r-s| \geq 1} e^{-c(r-s)^{2}} d r \leq C,
$$

and the proof of $(5.8)$ is completed.

Proof of b). By using an argument analogous to a), it is enough to prove that

$$
\left\|\int_{0}^{\infty} f(s) \mathcal{K}_{\alpha, \sigma}(r, s) d \mu_{\alpha}(s)\right\|_{L^{\infty}\left((0, \infty), d \mu_{\alpha}\right)} \leq C\|f\|_{L^{p}\left((0, \infty), d \mu_{\alpha}\right)} .
$$

Now, by Hölder's inequality, the result will follow from (5.8) and the symmetry of the kernel $\mathcal{K}_{\alpha, \sigma}$, by using the condition $\frac{1}{p}<\frac{\sigma}{\alpha+1}$.

Proof of $c$ ). We consider $r \in(0,2)$ and $r \geq 2$ separately. In the first case, by Proposition 5.1 and Lemma 4.5 , the inequality is reduced to prove

$$
\left\|\chi_{(0,2)}(r) \int_{0}^{\infty} f(s) \mathcal{K}_{\alpha, \sigma}(r, s) d \mu_{\alpha}(s)\right\|_{L^{q}\left((0, \infty), d \mu_{\alpha}\right)} \leq C\|f\|_{L^{\infty}\left((0, \infty), d \mu_{\alpha}\right)} .
$$

Now, taking into account that

$$
\int_{0}^{\infty} f(y) \mathcal{K}_{\alpha, \sigma}(r, s) d \mu_{\alpha}(s) \leq\|f\|_{L^{\infty}\left((0, \infty), d \mu_{\alpha}\right)} \int_{0}^{\infty} \mathcal{K}_{\alpha, \sigma}(r, s) d \mu_{\alpha}(s),
$$

we will conclude by showing that

$$
\left\|\chi_{(0,2)}(r) \int_{0}^{\infty} \mathcal{K}_{\alpha, \sigma}(r, s) d \mu_{\alpha}(s)\right\|_{L^{q}\left((0, \infty), d \mu_{\alpha}\right)} \leq C,
$$

but this is true by (5.8) with $q=1$.

When $r \geq 2$, by (5.6) and Lemma 4.5, it will be enough to prove that

$$
\left\|\chi_{[2, \infty)}(r) \int_{0}^{\infty} f(s) \overline{\mathcal{K}}_{\alpha, \sigma}(r, s) d \mu_{\alpha}(s)\right\|_{L^{q}\left((0, \infty), d \mu_{\alpha}\right)} \leq C\|f\|_{L^{\infty}\left((0, \infty), d \mu_{\alpha}\right)}
$$

By applying (5.7), we obtain that

$$
\begin{aligned}
\int_{2}^{\infty}\left(\int_{0}^{\infty} f(s) \overline{\mathcal{K}}_{\alpha, \sigma}(r, s) d \mu_{\alpha}(s)\right)^{q} d \mu_{\alpha}(r) & \leq\|f\|_{L^{\infty}\left((0, \infty), d \mu_{\alpha}\right)}^{q} \int_{2}^{\infty}\left(\int_{0}^{\infty} \overline{\mathcal{K}}_{\alpha, \sigma}(r, s) d \mu_{\alpha}(s)\right)^{q} d \mu_{\alpha}(r) \\
& \leq C\|f\|_{L^{\infty}\left((0, \infty), d \mu_{\alpha}\right)}^{q} \int_{2}^{\infty} r^{2 \alpha+1-2 \sigma q} d r \leq C\|f\|_{L^{\infty}\left((0, \infty), d \mu_{\alpha}\right)}^{q}
\end{aligned}
$$

where in the last step we have used the restriction $\frac{1}{q}<\frac{\sigma}{\alpha+1}$.

Proof of $d$ ). We distinguish between $s \in(0,2)$ and $s \geq 2$. In the first case, by Proposition 5.1 and Lemma 4.5, the inequality is reduced to prove

$$
\left\|\int_{0}^{2} f(s) \mathcal{K}_{\alpha, \sigma}(r, s) d \mu_{\alpha}(s)\right\|_{L^{1}\left((0, \infty), d \mu_{\alpha}\right)} \leq C\|f\|_{L^{p}\left((0, \infty), d \mu_{\alpha}\right)} .
$$

By Fubini's theorem and Hölder's inequality, we have

$$
\begin{aligned}
\int_{0}^{\infty} \int_{0}^{2} f(s) \mathcal{K}_{\alpha, \sigma}(r, s) d \mu_{\alpha}(s) d \mu_{\alpha}(r) & \leq\|f\|_{L^{p}\left((0, \infty), d \mu_{\alpha}\right)} \int_{0}^{2}\left\|\mathcal{K}_{\alpha, \sigma}(\cdot, s)\right\|_{L^{p^{\prime}\left((0, \infty), d \mu_{\alpha}\right)}} d \mu_{\alpha}(s) \\
& \leq C\|f\|_{L^{p}\left((0, \infty), d \mu_{\alpha}\right)}
\end{aligned}
$$

where in the last step we used (5.8). 
In the case $s \geq 2$, by (5.6) and Lemma 4.5, it will be enough to prove that

$$
\left\|\int_{2}^{\infty} f(s) \overline{\mathcal{K}}_{\alpha, \sigma}(r, s) d \mu_{\alpha}(s)\right\|_{L^{1}\left((0, \infty), d \mu_{\alpha}\right)} \leq C\|f\|_{L^{p}\left((0, \infty), d \mu_{\alpha}\right)} .
$$

Applying Fubini's theorem, Hölder's inequality and (5.7), we obtain

$$
\begin{aligned}
\int_{0}^{\infty} \int_{2}^{\infty} f(s) \overline{\mathcal{K}}_{\alpha, \sigma}(r, s) d \mu_{\alpha}(s) d \mu_{\alpha}(r) & \leq\|f\|_{L^{p}\left((0, \infty), d \mu_{\alpha}\right)}\left\|\chi_{(2, \infty)}(s) \int_{0}^{\infty} \overline{\mathcal{K}}_{\alpha, \sigma}(r, s) d \mu_{\alpha}(r)\right\|_{L^{p^{\prime}}\left((0, \infty), d \mu_{\alpha}\right)} \\
& \leq C\|f\|_{L^{p}\left((0, \infty), d \mu_{\alpha}\right)}\left\|\chi_{(2, \infty)}(s) s^{-2 \sigma}\right\|_{L^{p^{\prime}\left((0, \infty), d \mu_{\alpha}\right)}} \leq C\|f\|_{L^{p}\left((0, \infty), d \mu_{\alpha}\right)}
\end{aligned}
$$

where in the last step we used that $1-\frac{\sigma}{\alpha+1}<\frac{1}{p} \leq 1$.

Proof of e). By using Proposition 5.1 we have to distinguish three cases in terms of $\sigma$.

Case $\sigma<1 / 2$. In this case

$$
u_{j}(r)\left(L_{\alpha+a j}\right)^{-\sigma}\left(u_{j}^{-1}(\cdot) f_{j}\right)(r) \leq C\left(I_{1} f(r)+I_{2} f(r)+I_{3} f(r)\right)
$$

where

$$
I_{1} f(r)=\chi_{[0,2]}(r) r^{-2(\alpha+1-\sigma)} \int_{0}^{r / 2} f(s) d \mu_{\alpha}(s)+\chi_{[0,2]}(r) \int_{3 r / 2}^{2} s^{2 \sigma-1} f(s) d s,
$$

$I_{2} f(r)=L_{2 \sigma}^{\text {loc }} f(r)$, and

$$
I_{3} f(r)=\int_{|r-s| \geq 1} \frac{e^{-c(r-s)^{2}}}{(r+s)^{2 \alpha+1}} f(s) d \mu_{\alpha}(s) .
$$

For $I_{1}$ the required bound follows from Theorem 5.8, taking into account also the condition $\frac{1}{p}-\frac{\sigma}{\alpha+1}=\frac{1}{q}$. Indeed, for the first summand we are applying Theorem 5.8 with $\gamma=2(\alpha+$ $1-\sigma)-\frac{2 \alpha+2}{q}-1$, and for the second summand we apply Theorem 5.8 with $\gamma=-\frac{2 \alpha+2}{q}-1$. Concerning $I_{2}$, the estimate is an immediate consequence of Theorem 5.9. For $I_{3}$, we can prove the boundedness for the pairs $(1 / q, 1 / p)=(1-\sigma /(\alpha+1), 1)$ and $(1 / q, 1 / p)=(0, \sigma /(\alpha+1))$ as we did in a) and c). Indeed, the result will follow by applying Minkowski's or Hölder's inequalities and taking into account that

$$
\int_{|r-s| \geq 1} \frac{e^{-c(r-s)^{2}}}{(r+s)^{u(2 \alpha+1)}} d \mu_{\alpha}(s) \leq C,
$$

for any $u \geq 1$. The complete result is obtained by interpolation.

Case $\sigma=1 / 2$. Now, it is verified that $1 / p-1 / q=1 / 2(\alpha+1)=: \delta<1$. Then (since $-\log t \leq C t^{1-\delta}$, for $0<t<C<1)$

$$
u_{j}(r)\left(L_{\alpha+a j}\right)^{-\sigma}\left(u_{j}^{-1}(\cdot) f_{j}\right)(r) \leq C\left(M_{1} f(r)+M_{2} f(r)+I_{3} f(r)\right)
$$

where

$$
M_{1} f(r)=\chi_{[0,2]}(r) r^{-2(\alpha+1)} \int_{0}^{r} f(s) d \mu_{\alpha}(s)+\chi_{[0,2]}(r) \int_{r}^{2} s^{2 \sigma-1} f(s) d s,
$$

$M_{2} f(r)=L_{\delta}^{\mathrm{loc}} f(r)$, and $I_{3}$ is as in the previous case. In order to obtain the estimate we proceed analogously as for $\sigma<1 / 2$ by applying Theorem 5.8 and Theorem 5.9.

Case $\sigma>1 / 2$. Now, we have

$$
u_{j}(r)\left(L_{\alpha+a j}\right)^{-\sigma}\left(u_{j}^{-1}(\cdot) f_{j}\right)(r) \leq C\left(J_{1} f(r)+J_{2} f(r)\right),
$$

where

$$
J_{1} f(r)=\chi_{[0,2]}(r) r^{-2(\alpha+1-\sigma)} \int_{0}^{r} f(s) d \mu_{\alpha}(s)+\chi_{[0,2]}(r) \int_{r}^{2} s^{2 \sigma-1} f(s) d s,
$$

and

$$
J_{2} f(r)=\int_{r+s \geq 1} \frac{e^{-c(r-s)^{2}}}{(r+s)^{2 \alpha+1}} f(s) d \mu_{\alpha}(s) .
$$


The operator $J_{1}$ is covered by the argument for $I_{1}$ in the case $\sigma<1 / 2$ and for $J_{2}$ we proceed analogously as for $I_{3}$ in the case $\sigma<1 / 2$.

\section{Proofs of technical Results}

Proof of Lemma 5.2. First, observe that

$$
\log \left(\frac{1+\xi}{1-\xi}\right) \sim \begin{cases}\xi, & \text { for } 0<\xi \leq 1 / 2, \\ -\log \left(1-\xi^{2}\right), & \text { for } 1 / 2<\xi<1 .\end{cases}
$$

Then, denoting by $J$ the integral to be estimated, we have

$$
\begin{aligned}
J & \leq C \int_{0}^{1 / 2} \xi^{\sigma-c-\ell-1} \exp \left(-\frac{a}{4 \xi}\right) d \xi+C \int_{1 / 2}^{1}\left(-\log \left(1-\xi^{2}\right)\right)^{\sigma-1}\left(1-\xi^{2}\right)^{c} \xi^{-c-\ell} \exp \left(-\frac{a}{4 \xi}\right) d \xi \\
& =: J_{1}+J_{2} .
\end{aligned}
$$

Now, for $J_{1}$, the change of variable $s=\frac{a}{4 \xi}$ produces the required bound. Indeed,

$$
J_{1}=\frac{4^{c+\ell-\sigma}}{a^{c+\ell-\sigma}} \int_{\frac{a}{2}}^{\infty} e^{-s} s^{c+\ell-\sigma-1} d s \leq C \frac{4^{c} \Gamma(c+\ell-\sigma)}{a^{c+\ell-\sigma}} .
$$

In order to control $J_{2}$, we start by using the estimate

$$
t^{\gamma} e^{-t} \leq \gamma^{\gamma} e^{-\gamma}, \quad t, \gamma>0
$$

to deduce that

$$
\xi^{-c-\ell} \exp \left(-\frac{a}{4 \xi}\right) \leq \frac{4^{c+\ell-\sigma}}{a^{c+\ell-\sigma}} \xi^{-\sigma}(c+\ell-\sigma)^{c+\ell-\sigma} e^{-(c+\ell-\sigma)} .
$$

Then,

$$
\begin{aligned}
J_{2} & \leq \frac{4^{c+\ell-\sigma}}{a^{c+\ell-\sigma}}(c+\ell-\sigma)^{c+\ell-\sigma} e^{-(c+\ell-\sigma)} \int_{1 / 2}^{1}\left(-\log \left(1-\xi^{2}\right)\right)^{\sigma-1}\left(1-\xi^{2}\right)^{c} \xi^{-\sigma} d \xi \\
& \leq C \frac{4^{c+\ell-\sigma}}{a^{c+\ell-\sigma}}(c+\ell-\sigma)^{c+\ell-\sigma} e^{-(c+\ell-\sigma)} \int_{1 / 2}^{1}\left(-\log \left(1-\xi^{2}\right)\right)^{\sigma-1}\left(1-\xi^{2}\right)^{c} \xi d \xi .
\end{aligned}
$$

Now, after making the change of variable $1-\xi^{2}=e^{-t}$ in the second inequality below,

Therefore

$$
\begin{aligned}
\int_{1 / 2}^{1}\left(-\log \left(1-\xi^{2}\right)\right)^{\sigma-1}\left(1-\xi^{2}\right)^{c} \xi d \xi & \leq C \int_{1 / 2}^{1}\left(-\log \left(1-\xi^{2}\right)\right)^{\sigma-1 / 2}\left(1-\xi^{2}\right)^{c} \xi d \xi \\
& \leq \frac{C}{(c+1)^{\sigma+1 / 2}} \leq \frac{C}{(c+1)^{1 / 2}} .
\end{aligned}
$$

$$
J_{2} \leq C \frac{4^{c+\ell-\sigma}}{a^{c+\ell-\sigma}}(c+\ell-\sigma)^{c+\ell-\sigma-1 / 2} e^{-(c+\ell-\sigma)} .
$$

Finally, by Stirling's approximation, we conclude the bound for $J_{2}$.

Proof of Lemma 5.3. With the obvious bound

$$
\left(\frac{1-s}{A-B s}\right)^{\alpha+1 / 2} \leq \frac{1}{A^{\alpha+1 / 2}}
$$

we have

$$
I_{\alpha, b}^{\lambda} \leq \frac{1}{A^{\alpha+1 / 2}} \int_{0}^{1} \frac{(1-s)^{b-1}}{(A-B s)^{b+\lambda}} d s .
$$

Then, the change of variable $1-s=\frac{A-B}{B} z$ gives

$$
I_{\alpha, b}^{\lambda} \leq \frac{1}{A^{\alpha+1 / 2}} \frac{1}{B^{b}} \frac{1}{(A-B)^{\lambda}} \int_{0}^{\frac{B}{A-B}} \frac{z^{b-1}}{(1+z)^{b+\lambda}} d z .
$$


Now, for $\lambda>0$,

$$
\int_{0}^{\frac{B}{A-B}} \frac{z^{b-1}}{(1+z)^{b+\lambda}} d z \leq \int_{0}^{\infty} \frac{z^{b-1}}{(1+z)^{b+\lambda}} d z=\frac{\Gamma(b) \Gamma(\lambda)}{\Gamma(b+\lambda)} .
$$

In the case $\lambda=0$, we have

$$
\int_{0}^{\frac{B}{A-B}} \frac{z^{b-1}}{(1+z)^{b}} d z \leq \int_{0}^{\frac{B}{A-B}} \frac{1}{(1+z)} d z=\log \left(\frac{A}{A-B}\right) .
$$

Finally, for $\lambda<0$,

$$
\int_{0}^{\frac{B}{A-B}} \frac{z^{b-1}}{(1+z)^{b+\lambda}} d z \leq C \int_{0}^{\frac{A}{A-B}} z^{-\lambda-1} d z \sim\left(\frac{A-B}{A}\right)^{\lambda}
$$

Proof of Lemma 5.5. Due to the relation (5.1), it suffices to analyze the integral $J$ appearing in the proof of Proposition 4.2 with $\alpha+a j$ instead of $\alpha_{i}$ and in one dimension. So the integral to be analyzed is (we write here the letter $v$ for the variable of integration, for convenience)

$$
J:=\int_{-1}^{1} \exp \left(-\frac{q_{+}(r, s, v)}{4 \xi}-\frac{\xi q_{-}(r, s, v)}{4}\right)\left(1-v^{2}\right)^{\alpha+a j-1 / 2} d v .
$$

After the change of variable $v=2 u-1$ the integral becomes

$$
J=4^{\alpha+a j} \exp \left(-\frac{(r-s)^{2}}{4 \xi}-\frac{\xi(r+s)^{2}}{4}\right) \int_{0}^{1} \exp \left(-r s u\left(\frac{1}{\xi}-\xi\right)\right) u^{\alpha+a j-1 / 2}(1-u)^{\alpha+a j-1 / 2} d u .
$$

Now, it is easy to check that

$$
\begin{aligned}
J & \leq 4^{\alpha+a j+1 / 2} \exp \left(-\frac{(r-s)^{2}}{4 \xi}-\frac{\xi(r+s)^{2}}{4}\right) \int_{0}^{1 / 2} \exp \left(-r s u\left(\frac{1}{\xi}-\xi\right)\right) u^{\alpha+a j-1 / 2}(1-u)^{\alpha+a j-1 / 2} d u \\
& \leq C 4^{\alpha+a j+1 / 2} \exp \left(-\frac{(r-s)^{2}}{4 \xi}-\frac{\xi(r+s)^{2}}{4}\right) \int_{0}^{1 / 2} \exp \left(-r s u\left(\frac{1}{\xi}-\xi\right)\right) u^{\alpha+a j-1 / 2} d u
\end{aligned}
$$

Since $a j \geq 1$, the change of variable $\operatorname{rsu}\left(\frac{1}{\xi}-\xi\right)=z$ gives

$$
\begin{aligned}
J & \leq C 4^{\alpha+a j+1 / 2} \exp \left(-\frac{(r-s)^{2}}{4 \xi}-\frac{\xi(r+s)^{2}}{4}\right) \int_{0}^{1 / 2} \exp \left(-r s u\left(\frac{1}{\xi}-\xi\right)\right) u^{a j-1} d u \\
& \leq C 4^{\alpha+a j+1 / 2} \exp \left(-\frac{(r-s)^{2}}{4 \xi}-\frac{\xi(r+s)^{2}}{4}\right)(r s)^{-a j}\left(\frac{\xi}{1-\xi^{2}}\right)^{a j} \Gamma(a j) \\
& \leq C 4^{\alpha+a j+1 / 2} \exp \left(-\frac{(r-s)^{2}}{8 \xi}\right)\left|r^{2}-s^{2}\right|^{-2 \alpha-1}(r s)^{-a j}\left(\frac{\xi}{1-\xi^{2}}\right)^{a j} \Gamma(a j),
\end{aligned}
$$

where in the last step we have used the estimate

$$
\begin{aligned}
\exp \left(-\frac{(r-s)^{2}}{4 \xi}-\frac{\xi(r+s)^{2}}{4}\right) & \leq C \exp \left(-\frac{(r-s)^{2}}{8 \xi}\right) \exp \left(-c\left|r^{2}-s^{2}\right|\right) \\
& \leq C \exp \left(-\frac{(r-s)^{2}}{8 \xi}\right)\left|r^{2}-s^{2}\right|^{-2 \alpha-1}
\end{aligned}
$$

The result follows from (4.1), (4.4) and (5.1).

Proof of Proposition 5.6. From the identity $\left(L_{\alpha}\right)^{-\sigma}=\mathcal{I}_{\alpha, \sigma}$ in $L^{2}\left((0, \infty), d \mu_{\alpha}\right)$, the estimate in $(5.6)$ can be deduced from (5.2), (5.3), (5.1), and Proposition 4.2 (applied in one dimension).

In order to obtain the bound in (5.7) we start considering the case $|r-s|>r / 2$. Proceeding as in the proof of Proposition 4.3 with $n=1$, we have that the kernel $\overline{\mathcal{K}}_{\alpha, \sigma}$ can be estimated by $e^{-c(r-s)^{2}}(r s)^{-\alpha-1 / 2}$, then

$$
r^{2 \sigma} \int_{|r-s|>r / 2} \overline{\mathcal{K}}_{\alpha, \sigma}(r, s) d \mu_{\alpha}(s) \leq C \int_{|r-s|>r / 2}|r-s|^{2 \sigma-\alpha-1 / 2} e^{-c(r-s)^{2}} s^{\alpha+1 / 2} d s .
$$


If $0<s<r / 2$, we have

$$
\begin{aligned}
\int_{|r-s|>r / 2}|r-s|^{2 \sigma-\alpha-1 / 2} e^{-c(r-s)^{2}} s^{\alpha+1 / 2} d s & \leq C \int_{|r-s|>r / 2}|r-s|^{2 \sigma} e^{-c(r-s)^{2}} d s \\
& \leq C \int_{0}^{\infty} z^{2 \sigma} e^{-z^{2}} d z \leq C .
\end{aligned}
$$

When $s>3 r / 2$, it is verified that $|r-s| \sim s$, so that

$$
\int_{|r-s|>r / 2}|r-s|^{2 \sigma-\alpha-1 / 2} e^{-c(r-s)^{2}} s^{\alpha+1 / 2} d s \leq C \int_{0}^{\infty} s^{2 \sigma} e^{-c s^{2}} d s \leq C .
$$

In the most delicate region $|r-s| \leq r / 2$ we split the integral in $\overline{\mathcal{K}}_{\alpha, \sigma}$ into the intervals $(0,1 / 2)$ and $[1 / 2,1)$. For the second one, by using $(6.1)$, the integral of the kernel is controlled by

$$
r^{2 \sigma} e^{-c r^{2}} \int_{r / 2}^{3 r / 2} e^{-c(r-s)^{2}} \int_{1 / 2}^{1}\left(-\log \left(1-\xi^{2}\right)\right)^{\sigma-1}\left(1-\xi^{2}\right)^{-1 / 2} d \xi d s .
$$

This last integral is bounded because the inner integral is smaller than a constant and $r^{2 \sigma} e^{-c r^{2}} \leq C$.

In the case $\xi \in(0,1 / 2)$, by using again (6.1) and switching the order of integration we have

$$
\begin{aligned}
r^{2 \sigma} \int_{r / 2}^{3 r / 2} \int_{0}^{1 / 2} & \xi^{\sigma-3 / 2} \exp \left(-\frac{(r-s)^{2}}{4 \xi}-\frac{\xi r^{2}}{4}\right) d \xi d s \\
& =r^{2 \sigma} \int_{0}^{1 / 2} \xi^{\sigma-3 / 2} \exp \left(-\frac{\xi r^{2}}{4}\right) \int_{r / 2}^{3 r / 2} \exp \left(-\frac{(r-s)^{2}}{4 \xi}\right) d s d \xi \\
& \leq C r^{2 \sigma} \int_{0}^{1 / 2} \xi^{\sigma-1} \exp \left(-\frac{\xi r^{2}}{4}\right) d \xi \leq C r^{2 \sigma} r^{-2 \sigma}=C
\end{aligned}
$$

The proof is finished.

Acknowledgement. We are greatly indebted to José Luis Torrea for some fruitful discussions on the transference arguments among Laguerre families. We are also very grateful to the referees for the careful reading of the manuscript, and for their many remarks that helped to improve very much the paper.

\section{REFERENCES}

[1] I. Abu-Falahah, R. A. Macías, C. Segovia, and J. L. Torrea, Transferring strong boundedness among Laguerre orthogonal systems, Proc. Indian Acad. Sci. Math. Sci. 119 (2009), 203-220.

[2] P. Balodis and A. Córdoba, The convergence of multidimensional Fourier-Bessel series, J. Anal. Math. 77 (1999), 269-286.

[3] A. Benedek and R. Panzone, The space $L^{p}$ with mixed norm, Duke Math. J. 28 (1961), 301-324.

[4] B. Bongioanni and J. L. Torrea, Sobolev spaces associed to the harmonic oscillator, Proc. Indian Acad. Sci. Math. Sci. 116 (2006), 1-24.

[5] B. Bongioanni and J. L. Torrea, What is a Sobolev space for the Laguerre function systems?, Studia Math. 192 (2009), 147-172.

[6] A. Carbery, E. Romera, and F. Soria, Radial weights and mixed norm inequalities for the disc multiplier, J. Funct. Anal. 109 (1992), 52-75.

[7] Ó. Ciaurri and L. Roncal, The Riesz transform for the harmonic oscillator in spherical coordinates, Constr. Approx. 40 (2014), 447-472.

[8] Ó. Ciaurri, L. Roncal, and P. R. Stinga, Fractional integrals on compact Riemannian symmetric spaces of rank one, Adv. Math. 235 (2013), 627-647.

[9] A. Córdoba, The disc multiplier, Duke Math. J. 58 (1989), 21-29.

[10] K. Coulembier, H. De Bie, and F. Sommen, Orthogonality of Hermite polynomials in superspace and Mehler type formulae, Proc. Lond. Math. Soc. 103 (2011), 786-825.

[11] J. Duoandikoetxea, Fourier analysis, American Mathematical Society, Providence, 2001.

[12] J. Duoandikoetxea, Fractional integrals on radial functions with applications to weighted inequalities, Ann. Mat. Pura Appl. 192 (2013), 553-568.

[13] T. M. Flett, A note on some inequalities, Proc. Glasgow Math. Assoc. 4 (1958), 7-15. 
[14] J. García-Cuerva and J. L. Rubio de Francia, Weighted Norm Inequalities and Related Topics, North-Holland Mathematics Studies 16, North-Holland Publishing Co., Amsterdam, 1985.

[15] G. Garrigós, E. Harboure, T. Signes, J. L. Torrea, and B. Viviani, A sharp weighted transplantation theorem for Laguerre function expansions, J. Funct. Anal. 244 (2007), 247-276.

[16] L. Hörmander, Estimates for translation invariant operators in $L^{p}$ spaces, Acta Math. 104 (1960), 94-140.

[17] N. N. Lebedev, Special functions and its applications, Dover, New York, 1972.

[18] A. Nowak and L. Roncal, Potential operators associated with Jacobi and Fourier-Bessel expansions, J. Math. Anal. Appl. 422 (2015), 148-184.

[19] A. Nowak and K. Stempak, Riesz transforms for multi-dimensional Laguerre function expansions, Adv. Math. 215 (2007), 642-678.

[20] A. Nowak and K. Stempak, Negative powers of Laguerre operators, Canad. J. Math. 64 (2012), 183-216.

[21] A. Nowak and K. Stempak, Sharp estimates of the potential kernel for the harmonic oscillator with applications, Nagoya Math. J. 212 (2013), 1-17.

[22] A. Nowak and K. Stempak, Sharp estimates for potential operators associated with Laguerre and Dunkl-Laguerre expansions, Potential Anal. 44 (2016), 109-136.

[23] A. Nowak and K. Stempak, Potential operators and Laplace type multipliers associated with the twisted Laplacian, Acta Math. Sci. Ser. B Engl. Ed. 37 (2017), 280-292.

[24] J. L. Rubio de Francia, Transference principles for radial multipliers, Duke Math. J. 58 (1989), 1-19.

[25] E. Sasso, Functional calculus for the Laguerre operator, Math. Z. 249 (2005), 683-711.

[26] E. M. Stein, Singular integrals and differentiability properties of functions, Princeton Mathematical Series, Princeton University Press, Princeton, 1970.

[27] E. M. Stein and G. Weiss, Fractional integrals on $n$-dimensional Euclidean space, J. Math. Mech. 7 (1958), 503-514.

[28] P. R. Stinga and J. L. Torrea, Regularity theory for the fractional harmonic oscillator, J. Funct. Anal. 260 (2011), 3097-3131.

[29] S. Thangavelu, Lectures on Hermite and Laguerre expansions, Mathematical Notes 42, Princeton University Press, Princeton, 1993.

(Ó. Ciaurri) Departamento de Matemáticas y Computación, Universidad de La Rioja, 26006 Logroño, Spain E-mail address: oscar.ciaurri@unirioja.es

(L. Roncal) Departamento de Matemáticas y Computación, Universidad de La Rioja, 26006 Logroño, Spain. Current address: BCAM - Basque Center for Applied Mathematics, 48009 Bilbao, Spain

E-mail address: lroncal@bcamath.org 